\title{
Discontinuity-Detecting Method for a Four-Point Stencil and Its Application to Develop a Third-Order Hybrid-WENO Scheme
}

\author{
Shengping $\mathrm{Liu}^{1,2} \cdot$ Yiqing Shen ${ }^{1,3}$
}

Received: 8 November 2018 / Revised: 16 September 2019 / Accepted: 25 September 2019

(c) Springer Science+Business Media, LLC, part of Springer Nature 2019

\begin{abstract}
First this paper analyzes the reason for the accuracy losing of the third-order weighted essentially non-oscillatory (WENO) scheme. It is shown that one reason is that the local smoothness indicators of the third-order WENO scheme cannot correctly treat the smooth three-point stencil containing a non-nodal critical point, here, 'non-nodal' means the critical point is not a grid point. And then a discontinuity-detecting method for a four-point stencil is proposed and applied to develop the high order accurate hybrid-WENO scheme by combining the third-order WENO scheme and a third-order upstream scheme. This four-point stencil is actually the stencil used for constructing the third-order WENO scheme (positive and negative numerical fluxes), hence the resulting hybrid-WENO scheme proposed by this paper does not introduce new grid point. Numerical examples show that the detecting method and the hybrid scheme are robust for problems with shocks, and the hybrid scheme obtains real third-order convergence rate for smooth solutions containing critical points.
\end{abstract}

Keywords Third-order WENO scheme · Discontinuity-detecting method · Non-nodal critical point $\cdot$ Hybrid-WENO scheme

Mathematics Subject Classification 76M20 - 76L05

This research work was supported by the NKRDPC 2016YFA0401200, SCP No. TZ2016002, NSAF U1530145, NSFC Nos. 11872067 and 91852203.

Yiqing Shen

yqshen@imech.ac.cn

1 State Key Laboratory of High Temperature Gas Dynamics, Institute of Mechanics, Chinese Academy of Sciences, Beijing 100190, China

2 School of Aeronautic Science and Engineering, Beihang University, Beijing 100191, China

3 School of Engineering Science, University of Chinese Academy of Sciences, Beijing 100049, China 


\section{Introduction}

The weighted essentially non-oscillatory (WENO) scheme is first proposed by Liu et al. [1]. Its basic idea is to use a weighted convex combination of fluxes on all candidate sub-stencils instead of the one on the smoothest sub-stencil in ENO scheme [2]. By assigning adaptive weights to each sub-stencil, the WENO scheme can achieve high order accuracy in smooth regions while keeping the essentially non-oscillatory property near discontinuities. Jiang and Shu [3] introduced a general method for calculating smoothness indicators of stencils. Then, Balsara and Shu [4] extended the WENO schemes up to 11th order accuracy. Gerolymos et al. [5] further developed very-high-order WENO schemes. The fifth-order WENO scheme is the most widely studied and used in a number of references. Compared with higher order WENO schemes, the third-order one has several advantages [6]. For example, it is more robust for shock problems, it uses fewer grid points and hence it reduces the difficulty of boundary treatment and also it provides a suitable compromise of the computation cost and the accuracy in some applications. However, the numerical results show that the third-order WENO schemes always fail to obtain expected order near critical points. How to develop a real third-order accurate and low dissipative WENO scheme has attracted much attention.

Yamaleev and Carpenter [7] proposed the third-order energy stable WENO (ESWENO) scheme by combing the standard WENO scheme and an additional non-linear artificial dissipation term. Although the ESWENO scheme can achieve the optimal convergence order for a smooth solution, the two parameters, $\epsilon$ and $\delta$, designed for its weight function and the artificial dissipation term, are both dependent on the grid spacing (or the total number of grid cells), and $\epsilon$ is also determined by the initial solution. Later, Wu and Zhao [8] developed a less dissipative WENO-Z-type (WENO-N3) scheme by constructing a higher-order global smoothness indicator (GSI). Since the WENO-N3 scheme cannot obtain the optimal convergence order around critical points, Wu et al. [9] suggested using a power function of the GSI [8] as a new GSI to construct a new WENO-Z-type scheme (WENO-NP3) to avoid the loss of accuracy. Similar techniques are applied by Gande et al. [10] (WENO-NF3) and Xu and Wu [11] (WENO-NN3) to further improve the WENO-NP3 scheme. However, the modification of the GSI (i.e., WENO-NP3 and WENO-NF3) or local smoothness indicators (LSIs) (i.e., WENO-NN3) by using a power function destroys the requirement that the GSI and the LSIs should have the same dimension. Recently, $\mathrm{Xu}$ and $\mathrm{Wu}$ [12] modified the GSI of $\mathrm{Wu}$ et al. [8] and added a new term, which is similar to the one derived for a fifth-order WENO-Z scheme by Acker et al. [13], to increase the weight of less-smooth stencil and decrease the numerical dissipation of the third-order WENO-Z scheme. Although a parameter related to the grid spacing is also introduced in the added term, their scheme can not even reach second order convergence in $L_{\infty}$ norm.

Different from those works in [7-12], which focus on designing a higher-order GSI, Liu et al. [6] proposed a novel way to calculate the two local smoothness indicators(LSIs). As shown in [6], in monotonic smooth regions, the two LSIs are the same, thus the calculated weights are equal to the optimal ones, which means the resulting WENO (WENO-MN3) scheme recovers to the third-order upstream scheme. However, in non-monotonic smooth regions, i.e, around critical points, the two LSIs [6] have the similar behavior as that of the ones of the WENO-JS scheme, and hence the accuracy of the WENO-MN3 scheme is decreased.

In this paper, we firstly analyze the reason for decreasing accuracy of the third-order WENO schemes and it is shown that one reason is that the LSIs of the third-order WENO scheme cannot correctly distinguish the smooth three-point stencil containing a non-nodal 
critical point (i.e., the critical point is not a grid point) from discontinuous stencil. Then a discontinuity-detecting method for a four-point stencil is proposed and used to develop a third order accurate hybrid scheme of the third-order WENO scheme combining with a third-order upstream scheme.

This paper is arranged in the following way: in Sect. 2, the WENO-JS and WENO-MN3 schemes are briefly introduced, and the reason for decreasing accuracy of the third-order WENO schemes was analyzed. In Sect. 3, a discontinuity-detecting method for a four-point stencil is designed and applied to construct the third-order hybrid scheme. Section 4 gives numerical experiments. Section 5 is the conclusion remarks.

\section{Third-Order WENO Schemes}

In this section, we briefly introduce the WENO schemes by using the one-dimensional scalar conservation law equation [3],

$$
\frac{\partial u}{\partial t}+\frac{\partial f}{\partial x}=0
$$

where $u(x, t)$ is a conserved quantity, $f(u(x, t))$ describes its flux, and $x$ and $t$ denote space and time, respectively. By defining the points $x_{i}=i \Delta x,(i=0, \ldots, N)$, where $\Delta x$ is the uniform grid spacing, the Eq. (1) can be approximated by a conservative finite difference formula,

$$
\frac{d u_{i}}{d t}=-\frac{\hat{f}_{i+1 / 2}-\hat{f}_{i-1 / 2}}{\Delta x},
$$

where $\hat{f}_{i \pm 1 / 2}$ is the numerical flux. Generally, the flux can be split into positive part and negative part,

$$
\hat{f}_{i+1 / 2}=\hat{f}_{i+1 / 2}^{+}+\hat{f}_{i+1 / 2}^{-}
$$

Here, only the positive part $\hat{f}_{i+1 / 2}^{+}$is described, and the superscript " + " is dropped for simplicity. The negative part $\hat{f}_{i+1 / 2}^{-}$is symmetric with respect to $x_{i+1 / 2}$ and will not be shown.

\subsection{The WENO-JS and WENO-MN3 Schemes}

The flux $\hat{f}_{i+1 / 2}$ of a third-order WENO scheme can be written as

$$
\hat{f}_{i+1 / 2}=\omega_{0} q_{0}+\omega_{1} q_{1},
$$

where, $q_{k}$ is the second-order flux on the sub-stencil $S_{k}=(i-1+k, i+k)$ and is given by

$$
\left\{\begin{array}{l}
q_{0}=-\frac{1}{2} f_{i-1}+\frac{3}{2} f_{i}, \\
q_{1}=\frac{1}{2} f_{i}+\frac{1}{2} f_{i+1},
\end{array}\right.
$$

the weight $\omega_{k}$ is constructed as

$$
\omega_{k}=\frac{\alpha_{k}}{\alpha_{0}+\alpha_{1}}, \quad \alpha_{k}=\frac{c_{k}}{\left(I S_{k}+\epsilon\right)^{2}},
$$

where $c_{0}=1 / 3$ and $c_{1}=2 / 3$ are called the ideal weights. $\epsilon=10^{-40}$ is a small parameter introduced to avoid the denominator becoming zero. 
$I S_{k}$ in Eq. (6) is called as the local smoothness indicator that measures the smoothness of numerical solution on the sub-stencil $S_{k}$. In $[1,3], I S_{k}$ is calculated as

$$
\left\{\begin{array}{l}
I S_{0}=\left(f_{i}-f_{i-1}\right)^{2} \\
I S_{1}=\left(f_{i+1}-f_{i}\right)^{2} .
\end{array}\right.
$$

In smooth regions, the Taylor series expansions of Eq. (7) at $x_{i}$ give

$$
\left\{\begin{array}{l}
I S_{0}=\left(f_{i}^{\prime} \Delta x-\frac{1}{2} f_{i}^{\prime \prime} \Delta x^{2}+O\left(\Delta x^{3}\right)\right)^{2} \\
I S_{1}=\left(f_{i}^{\prime} \Delta x+\frac{1}{2} f_{i}^{\prime \prime} \Delta x^{2}+O\left(\Delta x^{3}\right)\right)^{2}
\end{array}\right.
$$

Hence, Eq. (8) can be written as

$$
I S_{k}=A(1+O(\Delta x)),
$$

where $A$ is independent of $k$. Substituting Eq. (9) into Eq. (6), there is

$$
\omega_{k}=c_{k}+O(\Delta x) \text {. }
$$

However, numerical experiments [6-8] showed that the WENO scheme (denoted by WENO-JS) with the weight function (Eq. 6) and indicators (Eq. 7) is too dissipative and can not even reach second order (the order of $L_{\infty}$ error) convergence.

In order to reduce the numerical dissipation of the third-order WENO-JS scheme, Liu et al. [6] proposed the following new indicators,

$$
\left\{\begin{array}{l}
I S_{0}^{N}=\frac{1}{4}\left(\left|f_{i+1}-f_{i-1}\right|-\left|4 f_{i}-3 f_{i-1}-f_{i+1}\right|\right)^{2} \\
I S_{1}^{N}=\frac{1}{4}\left(\left|f_{i+1}-f_{i-1}\right|-\left|3 f_{i+1}+f_{i-1}-4 f_{i}\right|\right)^{2}
\end{array} .\right.
$$

In smooth regions, the Taylor series expansions of Eq. (11) give

$$
\left\{\begin{array}{l}
I S_{0}^{N}=\left(\left|f_{i}^{\prime} \Delta x+O\left(\Delta x^{3}\right)\right|-\left|f_{i-1}^{\prime} \Delta x+O\left(\Delta x^{3}\right)\right|\right)^{2}, \\
I S_{1}^{N}=\left(\left|f_{i}^{\prime} \Delta x+O\left(\Delta x^{3}\right)\right|-\left|f_{i+1}^{\prime} \Delta x+O\left(\Delta x^{3}\right)\right|\right)^{2} .
\end{array}\right.
$$

Theoretically ( $\Delta x \rightarrow 0$ ), for a monotonic function, $f_{i-1}^{\prime}, f_{i}^{\prime}$ and $f_{i+1}^{\prime}$ have the same sign, hence, we can remove the absolute value bars in Eq. (11) and obtain

$$
I S_{0}^{N}=I S_{1}^{N}=\left(f_{i+1}-2 f_{i}+f_{i-1}\right)^{2} .
$$

Substituting Eq. (13) into the weight function Eq. (6) gives

$$
\omega_{0}=c_{0}, \quad \omega_{1}=c_{1},
$$

which means, in monotonic smooth regions, the weights are exactly equal to the optimal weights, i.e., the fully third-order upstream scheme is recovered. Due to this, the scheme is called WENO-MN3 [6].

For a discontinuous three-point stencil $\left(x_{i-1}, x_{i}, x_{i+1}\right)$, supposing $S_{0}=\left(x_{i-1}, x_{i}\right)$ is the discontinuous sub-stencil, and $S_{1}=\left(x_{i}, x_{i+1}\right)$ is the smooth one. Let

$$
\left\{\begin{array}{l}
\beta_{0}=f_{i}-f_{i-1} \\
\beta_{1}=f_{i+1}-f_{i} .
\end{array}\right.
$$


Since $\left|\beta_{0}\right|$ is much larger than $\left|\beta_{1}\right|$, i.e., $\left|\beta_{0}\right| \gg\left|\beta_{1}\right|$, the new indicators can be approximated by

$$
\left\{\begin{array}{l}
I S_{0}^{N}=\frac{1}{4}\left(\left|\beta_{0}+\beta_{1}\right|-\left|3 \beta_{0}-\beta_{1}\right|\right)^{2}=\left(\beta_{0}+\beta_{1}\right)^{2}=O\left(\beta_{0}^{2}\right) \\
I S_{1}^{N}=\frac{1}{4}\left(\left|\beta_{0}+\beta_{1}\right|-\left|3 \beta_{1}-\beta_{0}\right|\right)^{2}=\left(2 \beta_{1}\right)^{2}=O\left(\beta_{1}^{2}\right)
\end{array}\right.
$$

This shows that the new indicators (Eq. 11) approximate to the ones of Jiang and Shu's (Eq. 7). Hence, the ENO property can be maintained for problems with discontinuities. Please find more details in [6].

Although the WENO-MN3 scheme has less numerical dissipation than the WENO-JS scheme, the improvement of accuracy in $L_{\infty}$ norm of a smooth solution with critical points is still not remarkable [3].

\subsection{Analysis of the Accuracy of Third-Order WENO Schemes Around Critical Point}

In order to analyze the accuracy of a third-order WENO scheme around a critical point, by applying the Taylor expansion of $f$ at $x_{c}=x_{i}+\delta \Delta x(-1 \leq \delta \leq 1)$, we have

$$
\left\{\begin{array}{l}
f_{i-1}=f_{x_{c}}-(1+\delta) f_{x_{c}}^{\prime} \Delta x+\frac{1}{2}(1+\delta)^{2} f_{x_{c}}^{\prime \prime} \Delta x^{2}+O\left(\Delta x^{3}\right) \\
f_{i}=f_{x_{c}}-\delta f_{x_{c}}^{\prime} \Delta x+\frac{1}{2} \delta^{2} f_{x_{c}}^{\prime \prime} \Delta x^{2}+O\left(\Delta x^{3}\right) \\
f_{i+1}=f_{x_{c}}+(1-\delta) f_{x_{c}}^{\prime} \Delta x+\frac{1}{2}(1-\delta)^{2} f_{x_{c}}^{\prime \prime} \Delta x^{2}+O\left(\Delta x^{3}\right)
\end{array}\right.
$$

It is easy to find that

$$
\left\{\begin{array}{l}
I S_{0}=\left(f_{x_{c}}^{\prime} \Delta x+\frac{1}{2}(-1-2 \delta) f_{x_{c}}^{\prime \prime} \Delta x^{2}+O\left(\Delta x^{3}\right)\right)^{2} \\
I S_{1}=\left(f_{x_{c}}^{\prime} \Delta x+\frac{1}{2}(1-2 \delta) f_{x_{c}}^{\prime \prime} \Delta x^{2}+O\left(\Delta x^{3}\right)\right)^{2}
\end{array}\right.
$$

So, for a smooth solution with $f_{x_{c}}^{\prime}=0$, if and only if the coefficients of $f_{x_{c}}^{\prime \prime} \Delta x^{2}$ in $I S_{0}$ and I $S_{1}$ satisfy

$$
|-1-2 \delta|=|1-2 \delta|,
$$

the weights $\omega_{k}$ calculated by Eq. (6) can meet the relationship (Eq. 10). By solving Eq. (19), we can get $\delta=0$, which means the critical point $x_{c}$ is exactly the grid point $x_{i}$. That is, if there exists a critical point $x_{c}$ on the stencil $\left(x_{i-1}, x_{i}, x_{i+1}\right)$, the necessary condition for a third-order WENO scheme possibly getting higher order than the original ENO scheme is that the grid point $x_{i}$ is the critical point. It should be pointed out, the necessary condition also means that, for the case of $\delta= \pm 1$, i.e., the critical point $x_{c}$ is $x_{i \pm 1}$, the third-order WENO scheme can not improve the order yet.

Specially, if $\delta$ equals to $1 / 2$, we have

$$
\left\{\begin{array}{l}
I S_{0}=\left(f_{i+1 / 2}^{\prime \prime} \Delta x^{2}+O\left(\Delta x^{3}\right)\right)^{2}=O\left(\Delta x^{4}\right), \\
I S_{1}=O\left(\Delta x^{6}\right)
\end{array}\right.
$$

$I S_{1}$ is an infinitesimal with two order higher than $I S_{0}$, hence, this kind of three-points stencil $S^{3}=\left(x_{i-1}, x_{i}, x_{i+1}\right)$ may be quite possibly treated as a discontinuous stencil, and only first-order accuracy can be obtained.

According to the Taylor expansions in Sect. 2.1, theoretically, Eqs. (9) and (10) are always kept, even if $x_{i}$ is a critical point. Hence, by using the mapping function introduced by Henrick et al. [14],

$$
g_{k}\left(\omega_{k}\right)=\frac{\omega_{k}\left(c_{k}+c_{k}^{2}-3 c_{k} \omega_{k}+\omega_{k}^{2}\right)}{c_{k}^{2}+\omega_{k}\left(1-2 c_{k}\right)},
$$


the mapped weights should be

$$
\omega_{k}^{M}=c_{k}+O\left(\Delta x^{3}\right),
$$

which satisfy the sufficient condition for a third-order convergence of a third-order WENO scheme [6,7],

$$
\omega_{k}=c_{k}+O\left(\Delta x^{2}\right) .
$$

That means the mapped third-order WENO (WENO-M) scheme is strictly third-order accurate. However, the numerical results in [6] and also in this paper showed that the conclusion is not true. From the above analysis, it can be seen that, for the case of $f_{x_{c}}^{\prime}=0$ and $\delta \neq 0$, $I S_{0}$ and $I S_{1}$ have different coefficients of the term $f_{x_{c}}^{\prime \prime} \Delta x^{2}$, that means the condition Eq. (9) can not be satisfied, and hence Eq. (10) can not be reached. Suppose $x_{i}$ is a critical point on four-points stencil $\left(x_{i-2}, x_{i-1}, x_{i}, x_{i+1}\right)$, if the third-order WENO-M scheme is applied, then $\hat{f}_{i+1 / 2}$ (the three-points stencil $\left(x_{i-1}, x_{i}, x_{i+1}\right)$ is used) is a third-order flux, but $\hat{f}_{i-1 / 2}$ (the stencil $\left(x_{i-2}, x_{i-1}, x_{i}\right)$ is used) is not. Therefore, WENO-M can not achieve optimal third-order accuracy at critical point $x_{i}$.

Similarly, the indicators of WENO-MN3 (Eq. 11) can be analyzed by applying the following expansions,

$$
\left\{\begin{aligned}
I S_{0}^{N}= & \frac{1}{4}\left(\left|2 \delta f_{x_{c}}^{\prime \prime} \Delta x^{2}-\left(\frac{1}{3}+\delta^{2}\right) f_{x_{c}}^{\prime \prime \prime} \Delta x^{3}+O\left(\Delta x^{4}\right)\right|\right. \\
& \left.-\left|(2+2 \delta) f_{x_{c}}^{\prime \prime} \Delta x^{2}-\left(\frac{1}{3}+2 \delta+\delta^{2}\right) f_{x_{c}}^{\prime \prime \prime} \Delta x^{3}+O\left(\Delta x^{4}\right)\right|\right)^{2}, \\
I S_{1}^{N}= & \frac{1}{4}\left(\left|2 \delta f_{x_{c}}^{\prime \prime} \Delta x^{2}-\left(\frac{1}{3}+\delta^{2}\right) f_{x_{c}}^{\prime \prime \prime} \Delta x^{3}+O\left(\Delta x^{4}\right)\right|\right. \\
& \left.-\left|(2-2 \delta) f_{x_{c}}^{\prime \prime} \Delta x^{2}+\left(\frac{1}{3}-2 \delta+\delta^{2}\right) f_{x_{c}}^{\prime \prime \prime} \Delta x^{3}+O\left(\Delta x^{4}\right)\right|\right)^{2} .
\end{aligned}\right.
$$

We can see that, for cases of $\delta=0,-1,1, I S_{0}$ and $I S_{1}$ have the same coefficients of the term $\left(f_{x_{c}}^{\prime \prime} \Delta x^{2}\right)^{2}$. Hence, the WENO-MN3 scheme can deal well with those kinds of stencils, where $x_{i}$ or $x_{i-1}$ or $x_{i+1}$ is a critical point.

However, if $\delta \neq 0,-1,1$, i.e., the critical point $x_{c}$ is not a grid point, WENO-MN3 fails to improve the accuracy order. Specially, if $\delta=1 / 2$, there is

$$
\left\{\begin{aligned}
I S_{0}^{N}= & \frac{1}{4}\left(\left|f_{i+1 / 2}^{\prime \prime} \Delta x^{2}-\frac{28}{48} f_{i+1 / 2}^{\prime \prime \prime} \Delta x^{3}+O\left(\Delta x^{4}\right)\right|\right. \\
& \left.-\left|3 f_{i+1 / 2}^{\prime \prime} \Delta x^{2}-\frac{76}{48} f_{i+1 / 2}^{\prime \prime \prime} \Delta x^{3}+O\left(\Delta x^{4}\right)\right|\right)^{2}=O\left(\Delta x^{4}\right), \\
I S_{1}^{N}= & \frac{1}{4}\left(\left|f_{i+1 / 2}^{\prime \prime} \Delta x^{2}-\frac{28}{48} f_{i+1 / 2}^{\prime \prime \prime} \Delta x^{3}+O\left(\Delta x^{4}\right)\right|\right. \\
& \left.-\left|f_{i+1 / 2}^{\prime \prime} \Delta x^{2}-\frac{20}{48} f_{i+1 / 2}^{\prime \prime \prime} \Delta x^{3}+O\left(\Delta x^{4}\right)\right|\right)^{2}=O\left(\Delta x^{6}\right) .
\end{aligned}\right.
$$

In fact, for a series of discrete points of a continuous solution, $x_{i}$ is possibly a maximum point, but it is not a strict critical point (the first derivative is zero) of the solution. For convenience, we call this kind of critical point as non-nodal critical point. The above theoretical analysis shows that the local smoothness indicators cannot properly treat the smooth threepoint stencil containing a non-nodal critical point is one reason for the accuracy losing of the third-order WENO scheme.

Here, we use a function $u(x)=\sin (\pi x)$, which has a critical point at $x=0.5$, to illustrate the accuracy losing of third order WENO schemes numerically. Supposing the 
Table 1 Convergence rate of $\left.\frac{\partial u}{\partial x}\right|_{x_{i}}$, where, $x_{i}=x_{c}=0.5(\delta=0), x_{c}$ is a nodal point

\begin{tabular}{llllll}
\hline$\Delta x$ & $\begin{array}{l}\text { WENO-JS } \\
\text { Error (order) }\end{array}$ & $\begin{array}{l}\text { WENO-M } \\
\text { Error (order) }\end{array}$ & $\begin{array}{l}\text { WENO-MN3 } \\
\text { Error (order) }\end{array}$ & $\begin{array}{l}\text { WENO-NF3 } \\
\text { Error (order) }\end{array}$ & $\begin{array}{l}\text { Up3 } \\
\text { Error (order) }\end{array}$ \\
\hline 0.1 & $0.160(-)$ & $0.151(-)$ & $7.985 \mathrm{E}-3(-)$ & $1.004 \mathrm{E}-2(-)$ & $7.985 \mathrm{E}-3(-)$ \\
0.05 & $8.054 \mathrm{E}-2(0.99)$ & $7.611 \mathrm{E}-2(0.98)$ & $1.011 \mathrm{E}-3(2.98)$ & $1.319 \mathrm{E}-3(2.93)$ & $1.011 \mathrm{E}-3(2.98)$ \\
0.025 & $4.034 \mathrm{E}-2(1.00)$ & $3.816 \mathrm{E}-2(1.00)$ & $1.267 \mathrm{E}-4(3.00)$ & $1.670 \mathrm{E}-4(2.98)$ & $1.267 \mathrm{E}-4(3.00)$ \\
0.0125 & $2.018 \mathrm{E}-2(1.00)$ & $1.909 \mathrm{E}-2(1.00)$ & $1.585 \mathrm{E}-5(3.00)$ & $2.095 \mathrm{E}-5(3.00)$ & $1.585 \mathrm{E}-5(3.00)$ \\
\hline
\end{tabular}

Table 2 Convergence rate of $\left.\frac{\partial u}{\partial x}\right|_{x_{i}}$, where, $x_{i}=x_{c}-\delta \Delta x(\delta=1 / 2), x_{c}$ is a non-nodal critical point

\begin{tabular}{llllll}
\hline$\Delta x$ & $\begin{array}{l}\text { WENO-JS } \\
\text { Error (order) }\end{array}$ & $\begin{array}{l}\text { WENO-M } \\
\text { Error (order) }\end{array}$ & $\begin{array}{l}\text { WENO-MN3 } \\
\text { Error (order) }\end{array}$ & $\begin{array}{l}\text { WENO-NF3 } \\
\text { Error (order) }\end{array}$ & $\begin{array}{l}\text { Up3 } \\
\text { Error (order) }\end{array}$ \\
\hline 0.1 & $0.241 \mathrm{E}-1(-)$ & $0.612 \mathrm{E}-1(-)$ & $0.153(-)$ & $0.153(-)$ & $0.773 \mathrm{E}-2(-)$ \\
0.05 & $0.862 \mathrm{E}-2(1.48)$ & $0.269 \mathrm{E}-1(1.19)$ & $0.808 \mathrm{E}-1(0.92)$ & $0.808 \mathrm{E}-1(0.92)$ & $0.100 \mathrm{E}-2(2.95)$ \\
0.025 & $0.388 \mathrm{E}-2(1.15)$ & $0.130 \mathrm{E}-1(1.05)$ & $0.409 \mathrm{E}-1(0.98)$ & $0.409 \mathrm{E}-1(0.98)$ & $0.126 \mathrm{E}-3(2.99)$ \\
0.0125 & $0.189 \mathrm{E}-2(1.04)$ & $0.642 \mathrm{E}-2(1.01)$ & $0.205 \mathrm{E}-1(1.00)$ & $0.205 \mathrm{E}-1(1.00)$ & $0.158 \mathrm{E}-4(3.00)$ \\
\hline
\end{tabular}

stencil $S^{3}=(i-1, i, i+1)$ containing a critical point $\left(x_{c}=0.5\right)$, without loss of generality, let $x_{i}=x_{c}-\delta \Delta x$ (where $\delta$ is a parameter introduced to determine the location between the grid point $x_{i}$ and the critical point $x_{c},-1 \leq \delta \leq 1$ ), then we have

$$
\left\{\begin{array}{l}
u\left(x_{i-2}\right)=u\left(x_{i}-2 \Delta x\right) \\
u\left(x_{i-1}\right)=u\left(x_{i}-\Delta x\right) \\
u\left(x_{i}\right)=u\left(x_{i}\right) \\
u\left(x_{i+1}\right)=u\left(x_{i}+\Delta x\right)
\end{array}\right.
$$

Tables 1, 2 and 3 give the convergence rate of first derivative $\left.(\partial u / \partial x)\right|_{x=x_{i}}$ by using different schemes, where, the error is the absolute value of the difference of the numerical solution and the exact solution at $x_{i}$. As we can see, for the case of $\delta=0$ (nodal critical point), WENOMN3 get third-order accuracy, while WENO-JS and WENO-M are only first-order. For the cases with $\delta=1 / 2$ and $\delta=1 / 3$ (non-nodal critical point), the accuracy of WENO-JS, WENO-M and WENO-MN3 decreases to first order. These results are in agreement with the previous theoretical analysis.

The results of WENO-NF3 (see Eq. 44 in Sec. 4.1.1), are also given in Tables 1, 2 and 3. Numerical results show that, although WENO-NF3 can obtain third-order accuracy for cases of $\delta=0$ and $\delta=1 / 3$, it is incapable for $\delta=1 / 2$. In addition, since the power function of $\tau\left(\tau^{3 / 2}\right)$ is used to calculate the weights in WENO-NF3, this scheme may generate other issues, such as oscillation, non-similarity solutions (see Sects. 4.1.2 and 4.2).

\section{The New Method}

In this section, we introduce a discontinuity-detecting method for a four-point stencil and apply it to develop the third-order hybrid-WENO scheme. 
Table 3 Convergence rate of $\left.\frac{\partial u}{\partial x}\right|_{x_{i}}$, where, $x_{i}=x_{c}-\delta \Delta x(\delta=1 / 3), x_{c}$ is also a non-nodal critical point

\begin{tabular}{llllll}
\hline$\Delta x$ & $\begin{array}{l}\text { WENO-JS } \\
\text { Error (order) }\end{array}$ & $\begin{array}{l}\text { WENO-M } \\
\text { Error (order) }\end{array}$ & $\begin{array}{l}\text { WENO-MN3 } \\
\text { Error (order) }\end{array}$ & $\begin{array}{l}\text { WENO-NF3 } \\
\text { Error (order) }\end{array}$ & $\begin{array}{l}\text { Up3 } \\
\text { Error (order) }\end{array}$ \\
\hline 0.1 & $0.161 \mathrm{E}-1(-)$ & $0.429 \mathrm{E}-1(-)$ & $0.151(-)$ & $0.125 \mathrm{E}-1(-)$ & $0.784 \mathrm{E}-2(-)$ \\
0.05 & $0.573 \mathrm{E}-2(1.48)$ & $0.187 \mathrm{E}-1(1.20)$ & $0.794 \mathrm{E}-1(0.93)$ & $0.192 \mathrm{E}-2(2.71)$ & $0.101 \mathrm{E}-2(2.96)$ \\
0.025 & $0.258 \mathrm{E}-2(1.15)$ & $0.898 \mathrm{E}-2(1.06)$ & $0.402 \mathrm{E}-1(0.98)$ & $0.253 \mathrm{E}-3(2.92)$ & $0.127 \mathrm{E}-3(2.99)$ \\
0.0125 & $0.125 \mathrm{E}-2(1.04)$ & $0.445 \mathrm{E}-2(1.01)$ & $0.202 \mathrm{E}-1(1.00)$ & $0.321 \mathrm{E}-4(2.98)$ & $0.158 \mathrm{E}-4(3.00)$ \\
\hline
\end{tabular}

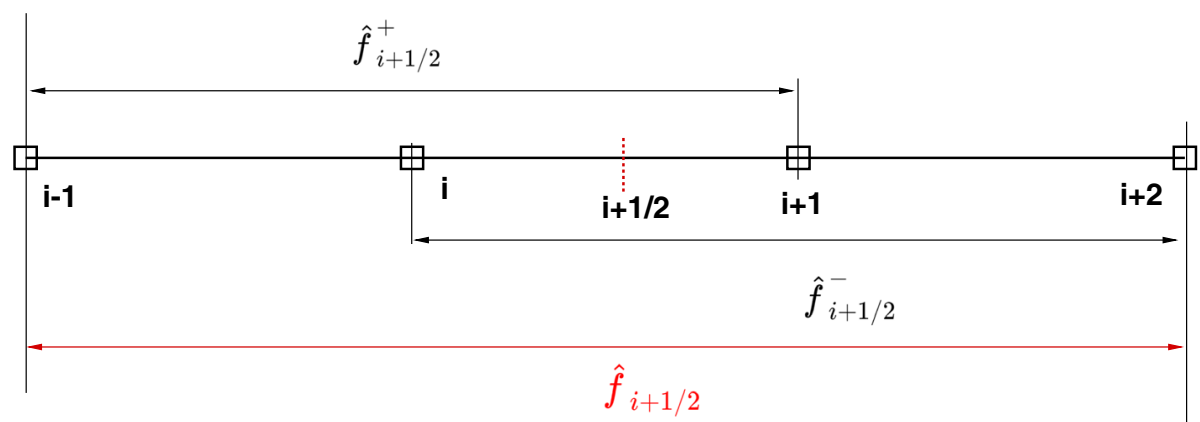

Fig. 1 The stencil of a third-order WENO scheme

\subsection{A Discontinuity-Detecting Method for a Four-Point stencil}

In Sect. 2, we illustrate theoretically that the third-order WENO scheme can not effectively distinguish that kind of three-point smooth stencils containing non-nodal critical points from discontinuous stencils. In order to overcome this shortcoming, it is necessary to develop a new method. As mentioned in Sect. 2, a complete formula of a WENO scheme contains positive and negative fluxes, and the two fluxes are symmetric with respect to $x_{i+1 / 2}$. For the third-order WENO scheme, the point $i+2$ is used in the negative flux (see Fig. 1). Hence, we hope adding point $i+2$ to the stencil $S^{3}=(i-1, i, i+1)$ to form a four-point stencil $S^{4}=(i-1, i, i+1, i+2)$, and use the stencil $S^{4}$ to help judge whether the stencil $S^{3}$ contains discontinuity or not.

Firstly, this paper proposes a discontinuity-detecting method for the four-point stencil $S^{4}$, i.e., if the condition

$$
\theta=\left|I C_{1}-I C_{2}\right| \geq \min \left(I C_{1}, I C_{2}\right)
$$

is satisfied, then the stencil $S^{4}=(i-1, i, i+1, i+2)$ can be regarded as a discontinuous stencil. Here, $I C_{1}$ is calculated as

$$
I C_{1}=\left(f_{i-1}-2 f_{i}+f_{i+1}\right)^{2},
$$

and it can be regarded as the smoothness indicator for the stencil $S^{3}=(i-1, i, i+1)$. Following the construction of $I S_{k}^{N}$ in WENO-MN3 [6], $I C_{2}$ is defined as

$$
I C_{2}=\frac{1}{4}\left(\left|f_{i+2}-f_{i}\right|-\left|3 f_{i+2}+f_{i}-4 f_{i+1}\right|\right)^{2},
$$


and can be regarded as the smoothness indicator for the sub-stencil $S_{2}=(i+1, i+2)$.

\subsection{The Performance of the Discontinuity-Detecting Method Eq. (27)}

First, supposing the stencil $S^{4}$ is a smooth stencil, and applying the Taylor expansion of $I C_{k}$ at $x_{c}\left(x_{c}=x_{i}+\delta \Delta x\right)$, there are

$$
\left\{\begin{aligned}
I C_{1}= & \left(f_{x_{c}}^{\prime \prime} \Delta x^{2}-\delta f_{x_{c}}^{\prime \prime \prime} \Delta x^{3}+O\left(\Delta x^{4}\right)\right)^{2} \\
= & \left(f_{x_{c}}^{\prime \prime}\right)^{2} \Delta x^{4}+O\left(\Delta x^{5}\right), \\
I C_{2}= & \left(\left|f_{x_{c}}^{\prime} \Delta x+(1-\delta) f_{x_{c}}^{\prime \prime} \Delta x^{2}+\left(\frac{2}{3}-\delta+\delta^{2} / 2\right) f_{x_{c}}^{\prime \prime \prime} \Delta x^{3}+O\left(\Delta x^{4}\right)\right|\right. \\
& \left.-\left|f_{x_{c}}^{\prime} \Delta x+(2-\delta) f_{x_{c}}^{\prime \prime} \Delta x^{2}+\left(\frac{5}{3}-2 \delta+\delta^{2} / 2\right) f_{x_{c}}^{\prime \prime \prime} \Delta x^{3}+O\left(\Delta x^{4}\right)\right|\right)^{2} \\
= & \left(f_{x_{c}}^{\prime \prime} \Delta x^{2}+O\left(\Delta x^{3}\right)\right)^{2}=\left(f_{x_{c}}^{\prime \prime}\right)^{2} \Delta x^{4}+O\left(\Delta x^{5}\right),
\end{aligned}\right.
$$

and

$$
\theta=\left|I C_{1}-I C_{2}\right|=O\left(\Delta x^{5}\right) .
$$

That means, $\theta$ is an infinitesimal with one order of $\Delta x$ higher than $I C_{1}$ and $I C_{2}$. So, if stencil $S^{4}=(i-1, i, i+1, i+2)$ is smooth, there is

$$
\theta \ll \min \left(I C_{1}, I C_{2}\right) .
$$

It can be seen that, Eqs. (30) and (31) are independent to the first derivative. That means, if stencil $S^{4}$ is a smooth stencil (no matter whether it contains non-nodal critical point or not), (32) is always satisfied.

Conversely, if there is

$$
\theta>\min \left(I C_{1}, I C_{2}\right),
$$

then the stencil $S^{4}$ can be regarded as a non-smooth stencil (non-strictly, we can call it as a discontinuous stencil).

For the convenience of the analyses of Eq. (33), let

$$
\left\{\begin{array}{l}
\beta_{0}=\left(f_{i}-f_{i-1}\right), \\
\beta_{1}=\left(f_{i+1}-f_{i}\right), \\
\beta_{2}=\left(f_{i+2}-f_{i+1}\right) .
\end{array}\right.
$$

And then, $I C_{1}$ and $I C_{2}$ can be rewritten as

$$
\left\{\begin{array}{l}
I C_{1}=\left(\beta_{1}-\beta_{0}\right)^{2}, \\
I C_{2}=\frac{1}{4}\left(\left|\beta_{1}+\beta_{2}\right|-\left|3 \beta_{2}-\beta_{1}\right|\right)^{2} .
\end{array}\right.
$$

Now, supposing there is a discontinuity contained in stencil $S^{4}$. Without loss of generality, assuming the discontinuity is located on sub-stencil $S_{1}=(i, i+1)$ (the other cases can be analyzed similarly), hence, there are

$$
\left|\beta_{1}\right| \gg\left|\beta_{0}\right| \text { and }\left|\beta_{1}\right| \gg\left|\beta_{2}\right|,
$$

so we can get

$$
\left\{\begin{array}{l}
I C_{1}=O\left(\beta_{1}^{2}\right) \\
I C_{2}=\frac{1}{4}\left(\beta_{1}+\beta_{2}-\left(\beta_{1}-3 \beta_{2}\right)\right)^{2}=O\left(\beta_{2}^{2}\right) .
\end{array}\right.
$$




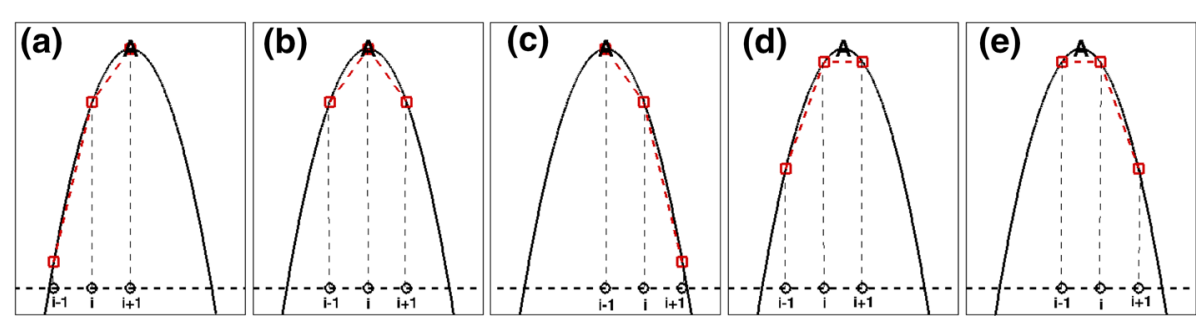

Fig. 2 Sketch of smooth stencils with three points

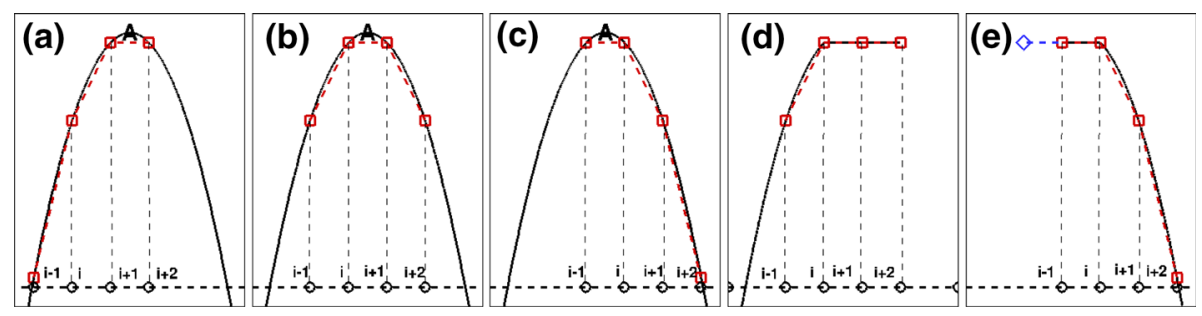

Fig. 3 Sketch of smooth stencils and discontinuous stencils with four points

Hence, there is

$$
\theta=\left|I C_{1}-I C_{2}\right| \simeq I C_{1} \gg I C_{2} .
$$

Above analysis shows that, if there is

$$
\theta<\min \left(I C_{1}, I C_{2}\right)
$$

the $S^{4}$ stencil should be a smooth stencil.

Now, let's discuss the smooth or discontinuous properties between the stencils $S^{3}$ and $S^{4}$.

(1) Stencil $S^{3}$ [for example, the cases (a)-(c) in Fig. 2] can be detected as a smooth stencil by using only the information on $S^{3}$.

The detecting method for a three-point stencil can be easily established following the $\tau_{5}$ detecting method for a five-point stencil proposed by Shen and Zha [15], if

$$
\tau_{3}^{N}=\left|I S_{0}^{N}-I S_{1}^{N}\right|<\min \left(I S_{k}^{N}\right)
$$

is satisfied, then $S^{3}$ can be treated as a smooth stencil.

(2) If $S^{3}$ is non-smooth [i.e., Eq. (40) is not satisfied], but $S^{4}$ is smooth, then $S^{3}$ can be treated as a smooth stencil containing a non-nodal critical point [see the three-point stencil of case (d) in Fig. 2 and the four-point stencil of case (b) in Fig. 3].

(3) The trouble situation is that, if both $S^{3}$ and $S^{4}$ are judged as non-smooth stencils, then how to treat the three-point stencil $S^{3}$. The trouble is caused by the two cases of (c) and (e) in Fig. 3. Because the $S^{3}$ of case (c) is a smooth stencil containing a non-nodal critical point, but the $S^{3}$ of case (e) (the blue 'diamond' indicates the node $i-2$ ) is actually a non-smooth stencil. Fortunately, the case (e) (in Fig. 2) is always paired with the case (d) (in Fig. 2), hence, in practical application, if step (2) is met, then the two neighbor three-point stencils can be simultaneously regarded as two smooth stencils containing non-nodal critical point; otherwise, i.e., if both $S^{3}$ and $S^{4}$ are judged as non-smooth stencils, then $S^{3}$ can be treated as a non-smooth stencil. 


\subsection{The Hybrid Scheme of WENO-MN3 and the Third-Order Scheme}

Based on the above analyses and conclusions, we can construct a hybrid algorithm on the three-point stencil $S^{3}=(i-1, i, i+1)$ by combining the WENO-MN3 $\left(\hat{f}_{i+1 / 2}^{W E N O-M N 3}\right)$ and the third-order upstream $\left(\hat{f}_{i+1 / 2}^{U p 3}\right)$ schemes. The algorithm is described as follows,

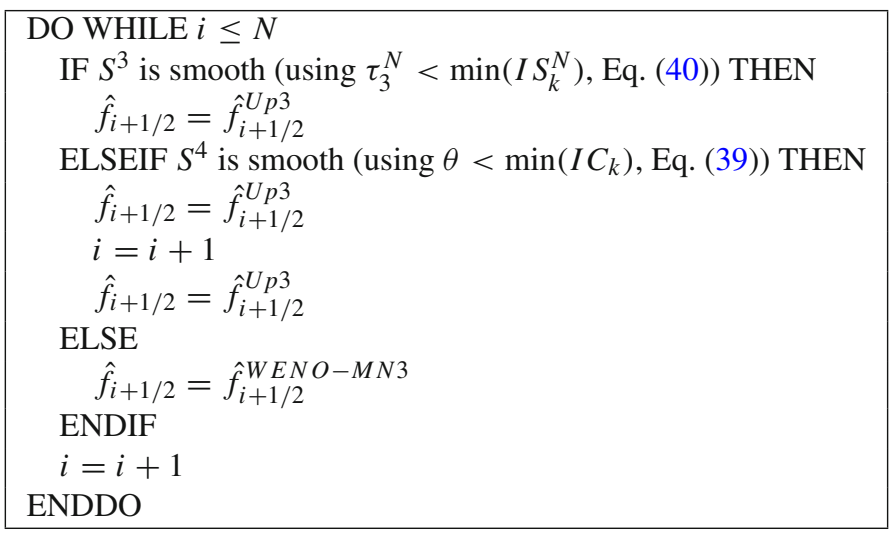

The hybrid scheme (denoted by HWMN3) can treat the stencils containing a non-nodal critical point (Fig. 3b, c) as smooth stencils, and it also can distinguish well the stencils containing a discontinuity (Fig. 3d, e).

In practical applications, we find that, treating the stencil of case (e) (in Fig. 2) as a discontinuous one, the influence on the accuracy of a numerical solution is not so apparent for most of the cases. In addition as the analyses shown in Sect. 2, the WENO-MN3 scheme can recovery to the Up3 scheme in monotonic smooth regions [where, the formula (40) is satisfied], hence, we also suggest a simplified HWMN3 scheme (denoted by S-HWMN3) as follows,

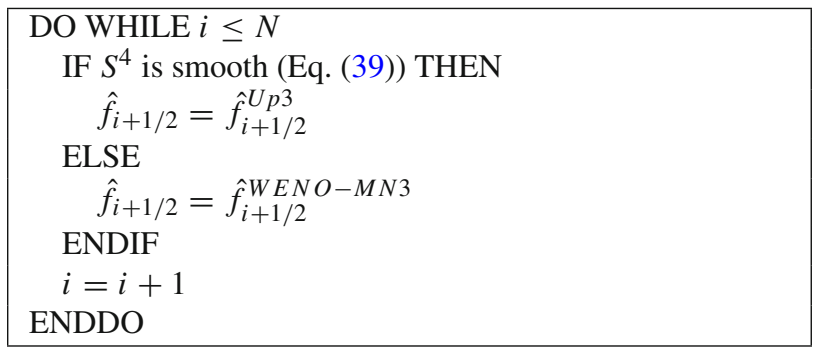

It is easy to find that, the S-HWMN3 scheme is less computational cost than HWMN3, since the $I S_{k}^{N}$ is calculated only if the stencil $S^{4}$ is judged as a discontinuous stencil.

\section{Numerical Examples}

In this section, several linear advection problems, one- (1-D) and two-dimensional (2-D) Euler problems are calculated to test the performance of the two hybrid schemes. Time advancement is performed with the third-order TVD Runge-Kutta method [16]. 


\subsection{Linear Advection Problems}

In the following we test the accuracy of different schemes on the linear advection equation,

$$
\begin{cases}\frac{\partial u}{\partial t}+\frac{\partial u}{\partial x}=0, & x_{0} \leqslant x \leqslant x_{1}, \\ u(x, t=0)=u_{0}(x), & \text { periodic boundary. }\end{cases}
$$

The exact solution of Eq. (41) is given by

$$
u(x, t)=u_{0}(x-t) .
$$

\subsubsection{Case 1}

$$
u_{0}(x)=\sin \left(\pi x-\frac{\sin \pi x}{\pi}\right), \quad-1 \leq x \leq 1 .
$$

This particular initial condition [14] has two critical points at which $f^{\prime}=0$ and $f^{\prime \prime} \neq 0$. It is used to test the accuracy of the hybrid scheme. The time step is chosen to be $\Delta t=$ $8 \Delta x^{5 / 3}$ in order that the error for the overall scheme is a measure of the spatial convergence only [14]. The errors and the convergence order in $L_{1}$ and $L_{\infty}$ norm at $t=2$ are given in Tables 4 and 5.

It is noticed that, WENO-NP3 [9], WENO-NF3 [10] and WENO-NN3 [11] have the similar form as that of WENO-Z-type scheme, i.e., the formula of their un-normalized weights $\alpha_{k}$ can be expressed as

$$
\alpha_{k}=c_{k}\left(1+\frac{\tau^{p}}{\left(I S_{k}+\epsilon\right)^{q}}\right),
$$

where, $\tau=\left|\frac{I S_{0}+I S_{1}}{2}-I S_{3}\right|=\gamma\left(f_{i-1}+f_{i+1}-2 f_{i}\right)^{2}$ (see [6,12] for more information). The corresponding parameters (i.e., $q, p$ and $\gamma$ ) for above mentioned schemes are given in Table 6.

WENO-NP3 and WENO-NF3 behave similarly as WENO-NN3, so their results do not show here. Apparently, both the HWMN3 and S-HWMN3 schemes recover to the full third order accuracy, which indicates the discontinuity-detecting method for $S^{4}$ stencil works well for smooth solutions. Although WENO-NN3 can obtain third order accuracy in $L_{1}$ norm, its $L_{\infty}$ order is only about second order. It should be pointed out that, the accuracy of the WENO-NP3, WENO-NF3 and WENO-NN3 schemes are affected by the time step, i.e., the $C F L$ number. To show this, Table 7 gives the results with $C F L=0.45$ (which is used in [10]) and $N=400$. It can be seen that, the error of the WENO-NF3 scheme even increases when a smaller time step is used. This is possible that, with a certain time step, the WENONF3 scheme can avoid or reduce the appearance of this kind of stencils containing non-nodal critical points.

Although these schemes (such as WENO-NF3) can possibly obtain good results with certain time steps, the main deficiency of them is that they destroy the requirement that the global smoothness indicator and the local smoothness indicators [the numerator and denominator in weighting formula (44)] should have the same dimension, and hence different solutions would be obtained if different reference values are chosen, even spurious solutions would be generated. This will be shown in Sects. 4.1.2 and 4.2. 


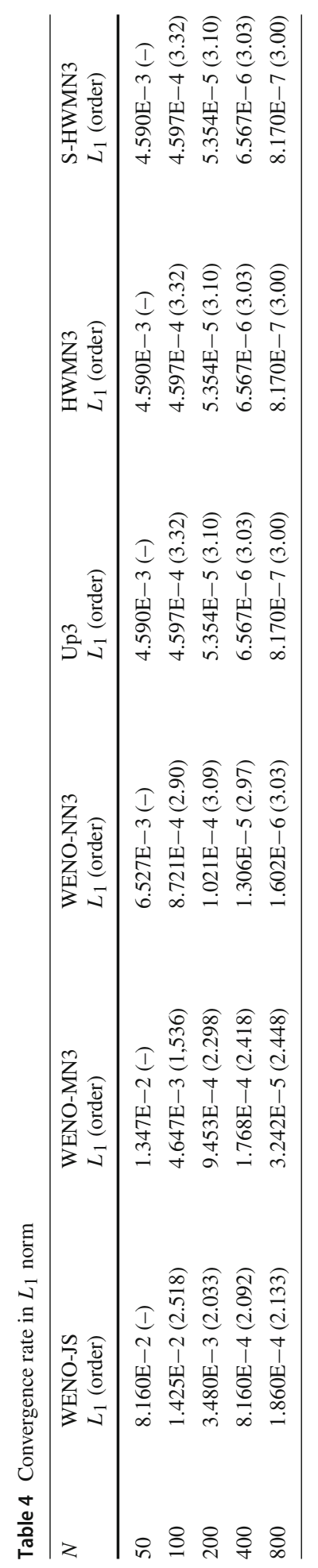




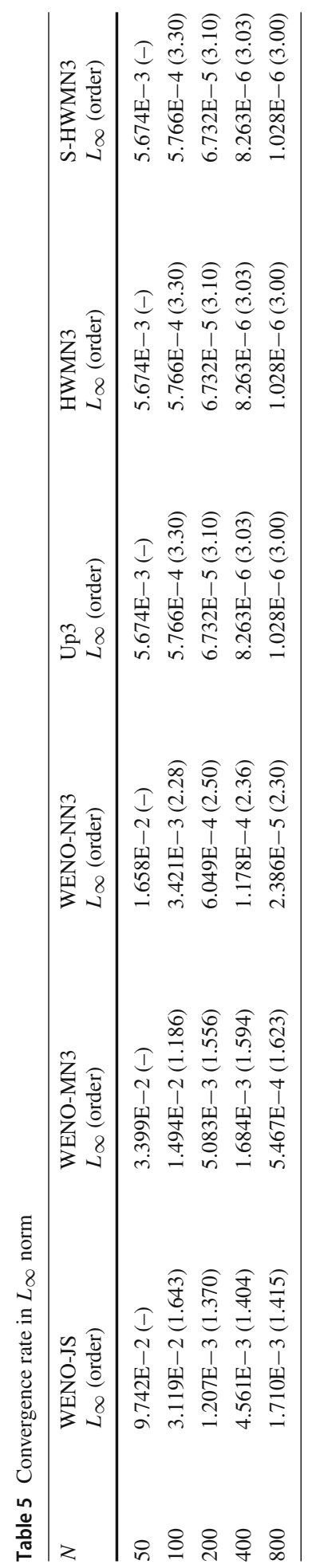


Table 6 The parameters of the WENO-NP3, WENO-NF3 and WENO-NN3 schemes

\begin{tabular}{llll}
\hline Scheme & $\gamma$ & $p$ & $q$ \\
\hline WENO-NP3 & $5 / 6$ & $3 / 2$ & 1 \\
WENO-NF3 & $1 / 6$ & $3 / 2$ & 1 \\
WENO-NN3 & $5 / 6$ & 1 & $3 / 4$ \\
\hline
\end{tabular}

Table 7 The errors in $L_{\infty}$ norm of WENO-NF3, Up3, HWMN3 and S-HWMN3 with different time steps

\begin{tabular}{|c|c|c|c|c|}
\hline Time step & WENO-NF3 & Up3 & HWMN3 & S-HWMN3 \\
\hline$\Delta t=8 \Delta x^{5 / 3}$ & $1.029 \mathrm{E}-5$ & $8.263 \mathrm{E}-6$ & $8.263 \mathrm{E}-6$ & $8.263 \mathrm{E}-6$ \\
\hline$\Delta t=0.45 \Delta x$ & $8.584 \mathrm{E}-6$ & $8.584 \mathrm{E}-6$ & $8.584 \mathrm{E}-6$ & $8.584 \mathrm{E}-6$ \\
\hline
\end{tabular}

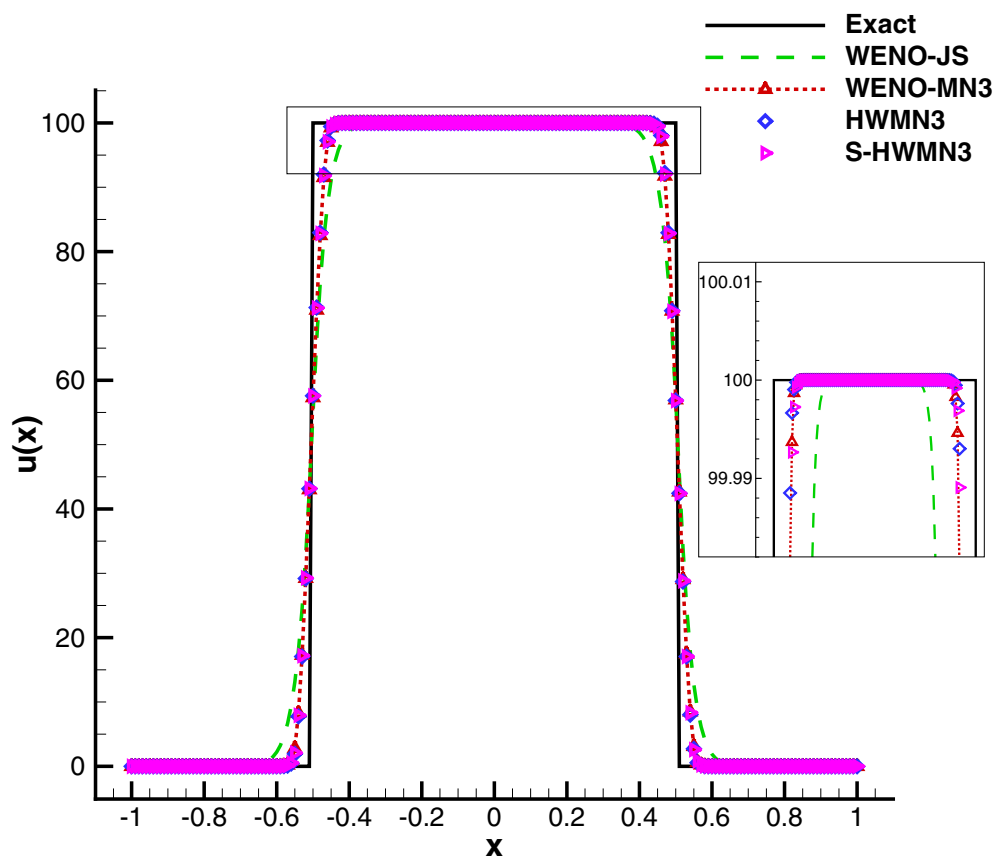

Fig. 4 Case 2, $A=100, t=2, N=200$, using the WENO-JS, WENO-MN3, HWMN3 and S-HWMN3 schemes

\subsubsection{Case 2}

$$
u_{0}(x)= \begin{cases}A, & \text { if }|x|<0.5 \\ 0, & \text { otherwise }\end{cases}
$$

where, $A$ is a constant number. This case is used to test the shock capturing capability and to show the importance of keeping the principle of similarity [17] of different schemes. The numerical solutions at $t=2$ with $N=200$ and time step $\Delta t=\Delta x / 2$ are shown in Figs. 4 , 5, 6 and 7. As these figures shown, the results computed by the WENO-JS, WENO-MN3, 


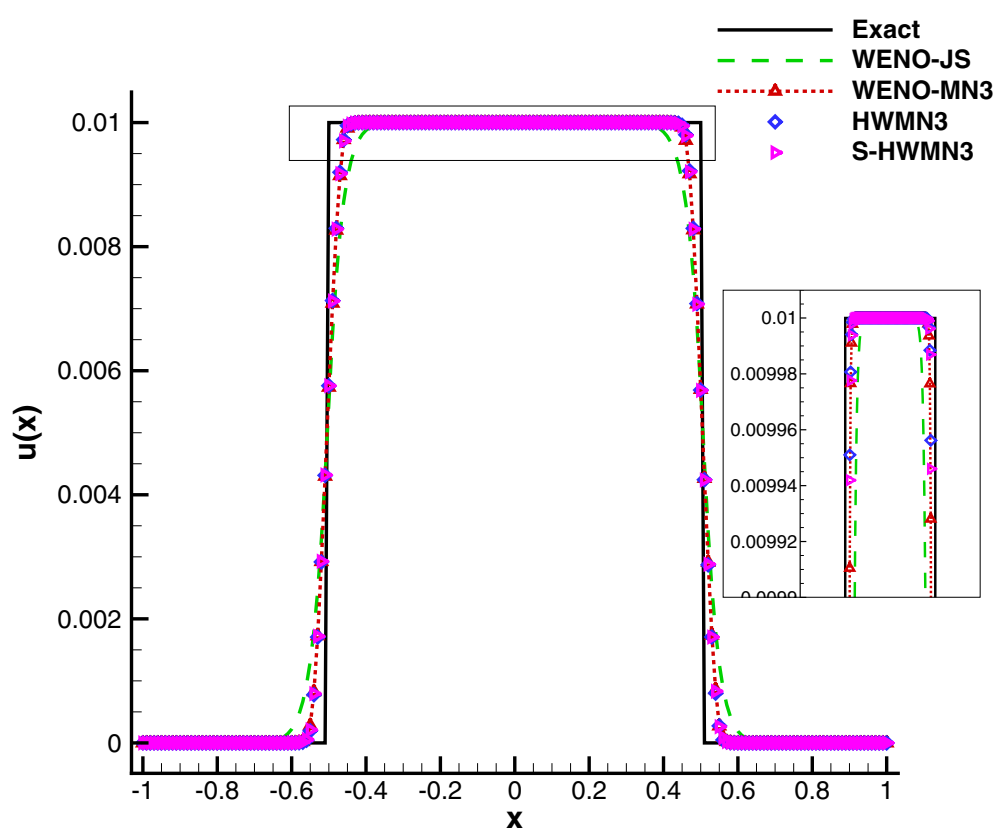

Fig. 5 Case 2, $A=0.01, t=2, N=200$, using the WENO-JS, WENO-MN3, HWMN3 and S-HWMN3 schemes

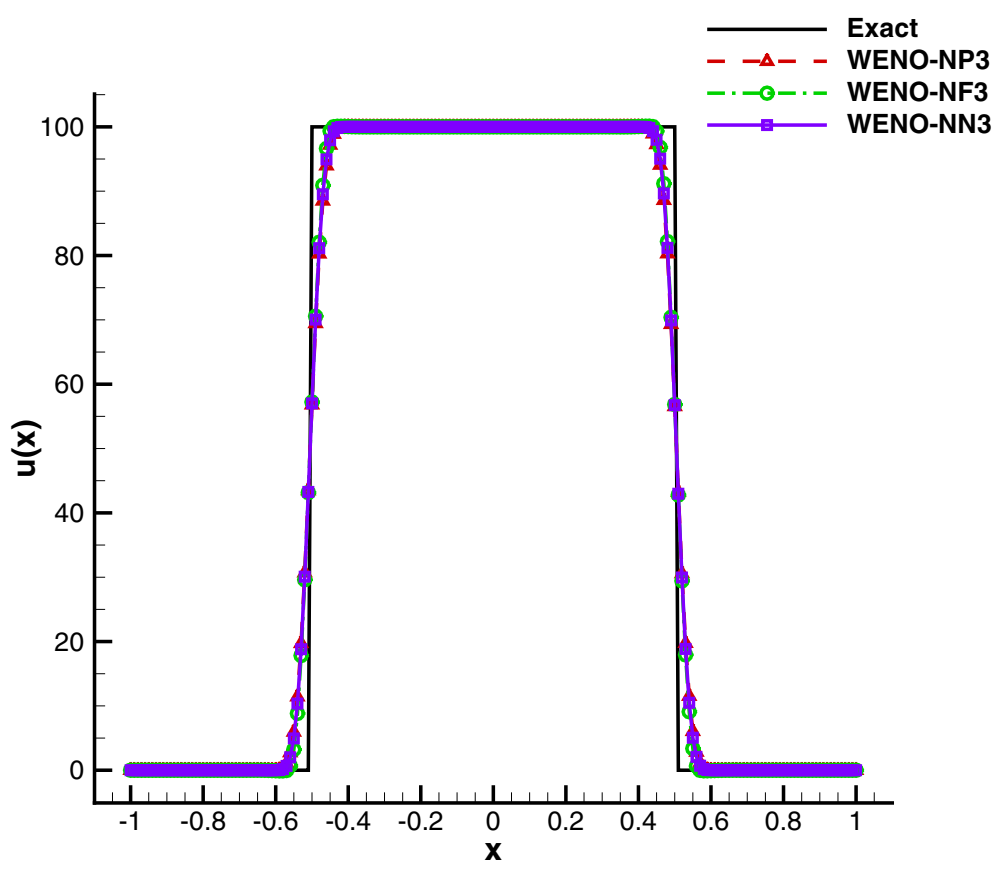

Fig. 6 Case 2, $A=100, t=2, N=200$, using the WENO-NP3, WENO-NF3 and WENO-NN3 schemes 


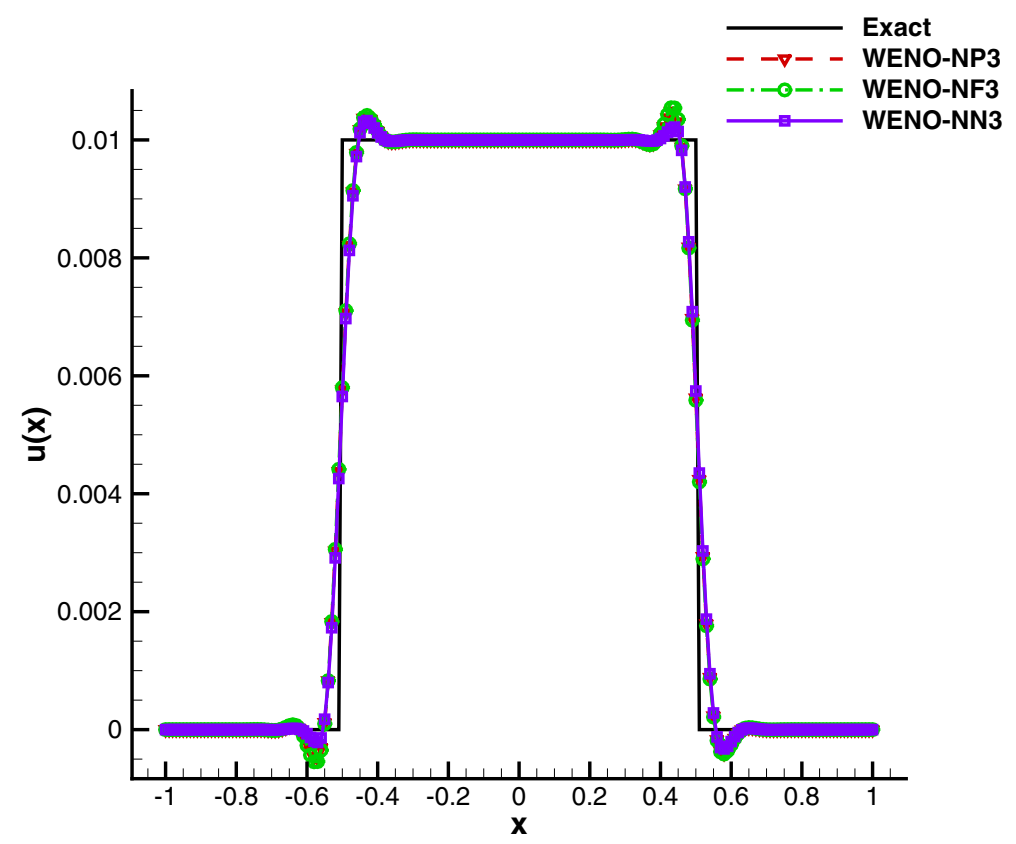

Fig. 7 Case 2, $A=0.01, t=2, N=200$, using the WENO-NP3, WENO-NF3 and WENO-NN3 schemes

HWMN3 and S-HWMN3 schemes with different values of $A$ (i.e., different reference values are used as the dimensionless parameter) behave similarly, and both the two hybrid schemes capture the discontinuities quite well.

However, the solutions obtained by the WENO-NP3, WENO-NF3 and WENO-NN3 schemes show different behaviors, for example, the solutions with $A=0.01$ (Fig. 7) are even oscillatory.

\subsubsection{Case 3}

$$
u_{0}(x)=e^{-(x-90)^{2} / 400}\left(\cos \left(\frac{\pi}{8}(x-90)\right)+\cos \left(\frac{\pi}{4}(x-90)\right)\right), \quad 50 \leq x \leq 130 .
$$

This case is used to test the performance of different schemes for a smooth solution containing several critical points [18]. Figures 8 and 9 show the numerical solutions with grid number $N=200$ and time step $\Delta t=\Delta x / 2$ at $t=80$ and $t=400$, respectively. As shown in these figures, the results of the Up3 and HWMN3 schemes are almost the same. It also can be seen that, in some regions, the accuracy of the S-HWMN3 scheme is decreased after several periods, since some three-point stencils [case (c) in Fig. 3] containing non-nodal critical points are treated as non-smooth stencils. 


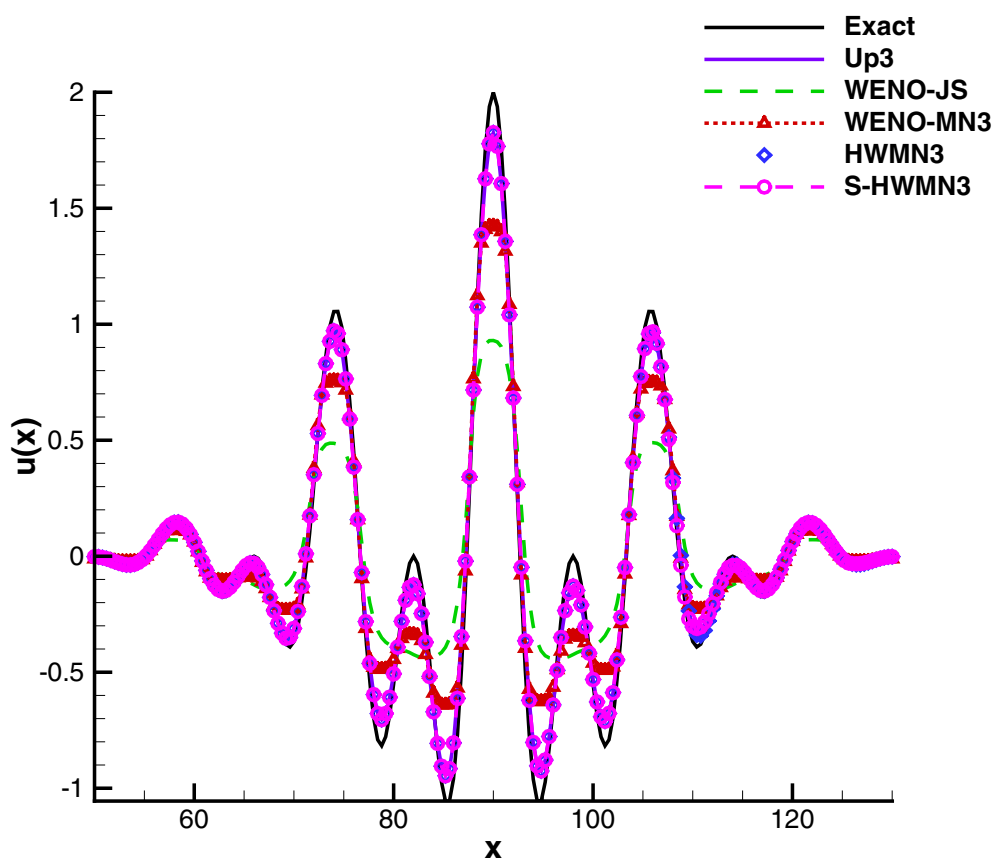

Fig. 8 Case 3, $t=80, N=200$, using the Up3, WENO-JS, WENO-MN3, HWMN3 and S-HWMN3 schemes

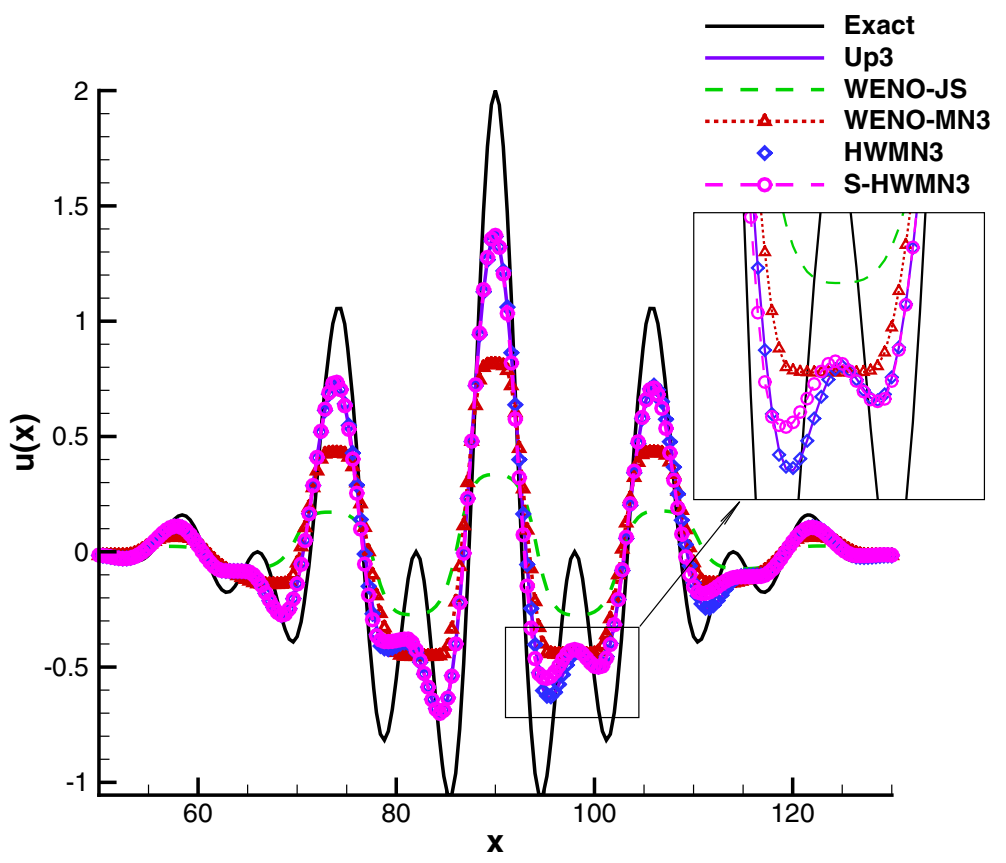

Fig. 9 Case $3, t=400, N=200$, using the Up3, WENO-JS, WENO-MN3, HWMN3 and S-HWMN3 schemes 


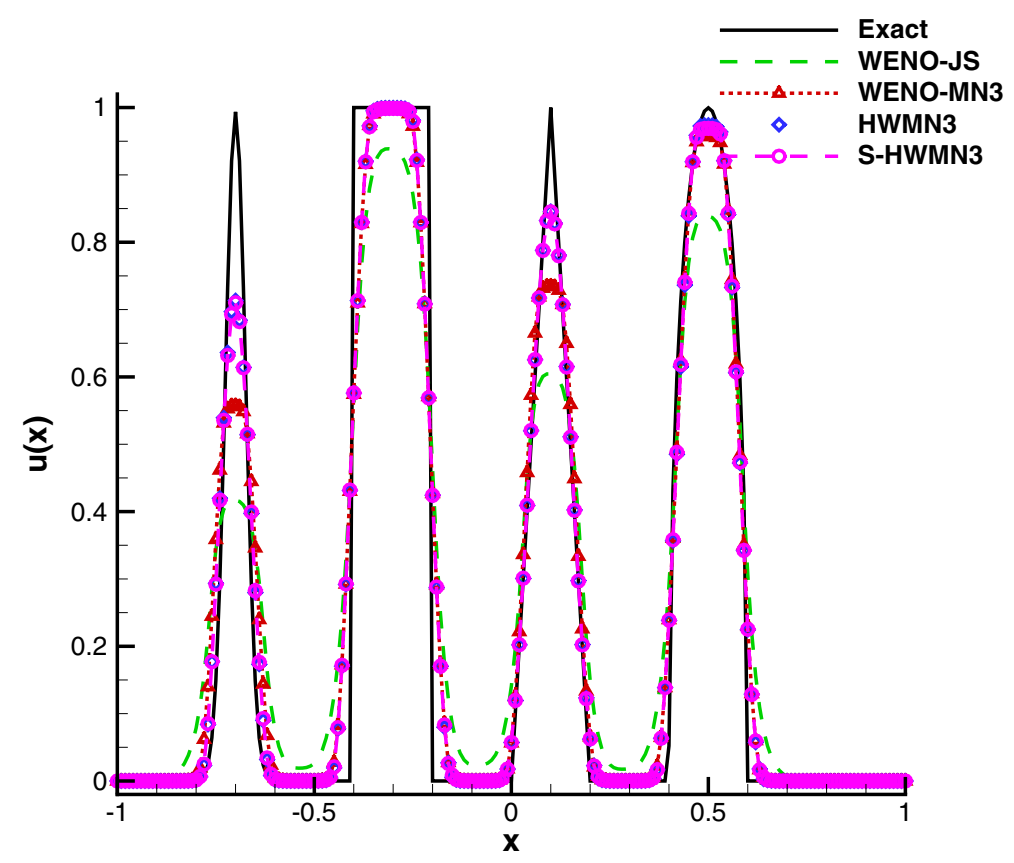

Fig. 10 Case 4, $t=2, N=200$, using the WENO-JS, WENO-MN3, HWMN3 and S-HWMN3 schemes

\subsubsection{Case 4}

$$
\begin{aligned}
u(x, 0) & = \begin{cases}\frac{1}{6}(G(x, \beta, z-\delta)+G(x, \beta, z+\delta)+4 G(x, \beta, z)), & -0.8 \leqslant x<-0.6, \\
1, & -0.4 \leqslant x<-0.2 \\
1-|10(x-0.1)|, & 0 \leqslant x<0.2 \\
\frac{1}{6}(F(x, \alpha, a-\delta)+F(x, \alpha, a+\delta)+4 F(x, \alpha, a)), & 0.4 \leqslant x<0.6 \\
0, & \text { otherwise }\end{cases} \\
G(x, \beta, z)=e^{-\beta(x-z)^{2}}, F(x, \beta, a)=\sqrt{\max \left(1-\alpha^{2}(x-a)^{2}, 0\right)} &
\end{aligned}
$$

This is a classical testing case, which contains a Gaussian, a triangle, a square-wave and a semi-ellipse. It is often used to test the dissipation of high-order schemes. The constants are $z=-0.7, \delta=0.005, \beta=\log (2) /\left(36 \delta^{2}\right), a=0.5$ and $\alpha=10$. It is solved with $N=200$ and time step $\Delta t=\Delta x / 2$. Figures 10 and 11 display the results at $t=2$ and $t=8$, respectively. Both the two hybrid schemes can capture discontinuities as well as WENO-MN3, they have less dissipation than WENO-JS and WENO-MN3, especially for the Gaussian, triangle and semi-ellipse waves. 


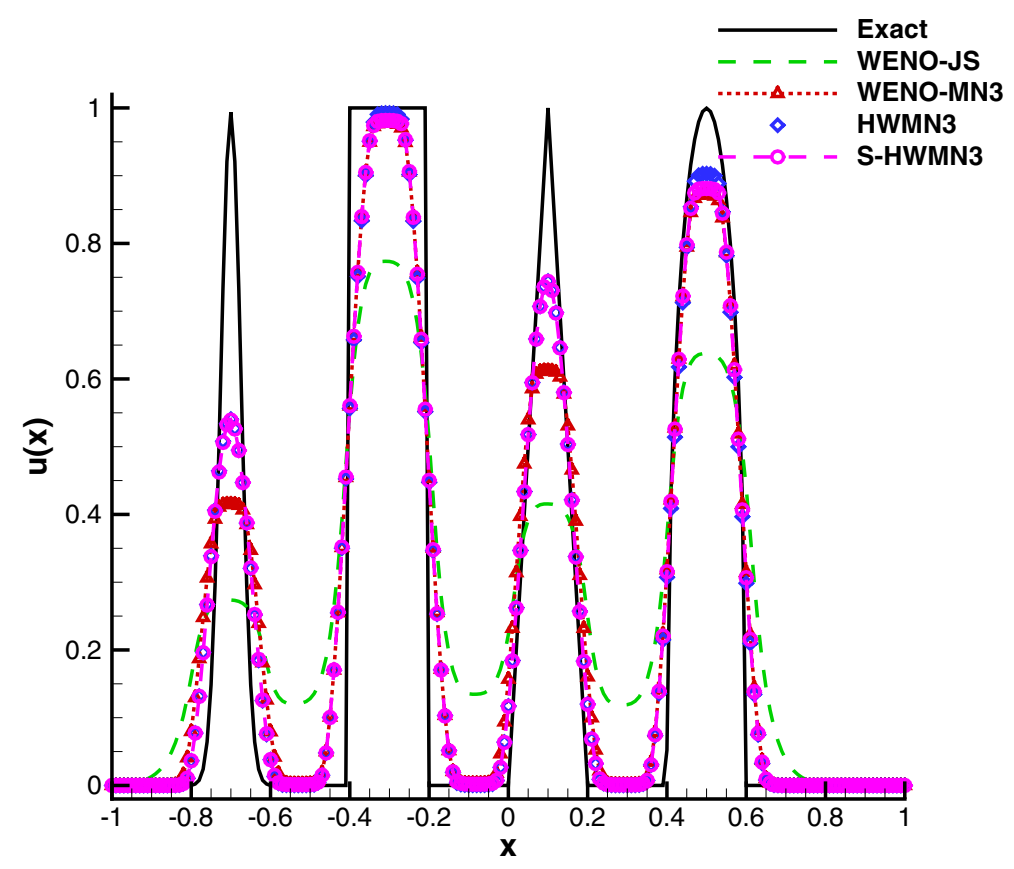

Fig. 11 Case 4, $t=8, N=200$, using the WENO-JS, WENO-MN3, HWMN3 and S-HWMN3 schemes

\subsection{One-Dimensional Euler Problems}

The governing equations are as follows,

$$
\frac{\partial U}{\partial t}+\frac{\partial F}{\partial x}=0
$$

where $U=(\rho, \rho u, E)^{T}, F(U)=\left(\rho u, \rho u^{2}+p, u(E+p)\right)^{T} . \rho, u, E, p$ are the density, velocity, total energy and pressure, respectively. For ideal gas, $E=p /(\gamma-1)+\rho u^{2} / 2$. $\gamma=1.4$ is the ratio of specific heat. Time step is taken as,

$$
\Delta t=\frac{\sigma \Delta x}{\max _{i}\left(\left|u_{i}\right|+c_{i}\right)},
$$

where, $\sigma=0.5$ is $C F L$ number, $c=\sqrt{\gamma p / \rho}$ is the speed of sound. The Characteristic-wise Roe-type decomposition method [19] is used for the local characteristic reconstruction. All the reference solutions are obtained by the WENO-JS scheme with 4000 points.

In order to show the possible drawback of the small parameter $\epsilon$, which is introduced to avoid the denominator becoming zero for calculating the weights, taken as a function of the grid spacing, in this section, we also give the results by using the weighted scheme with,

$$
\alpha_{k}=c_{k}\left(1+\frac{\tau}{I S_{k}+\Delta x^{2}}\right), \quad \tau=\left(f_{i-1}+f_{i+1}-2 f_{i}\right)^{2}
$$

In corresponding figures, the results are marked as $\epsilon=\Delta x^{2}$. 


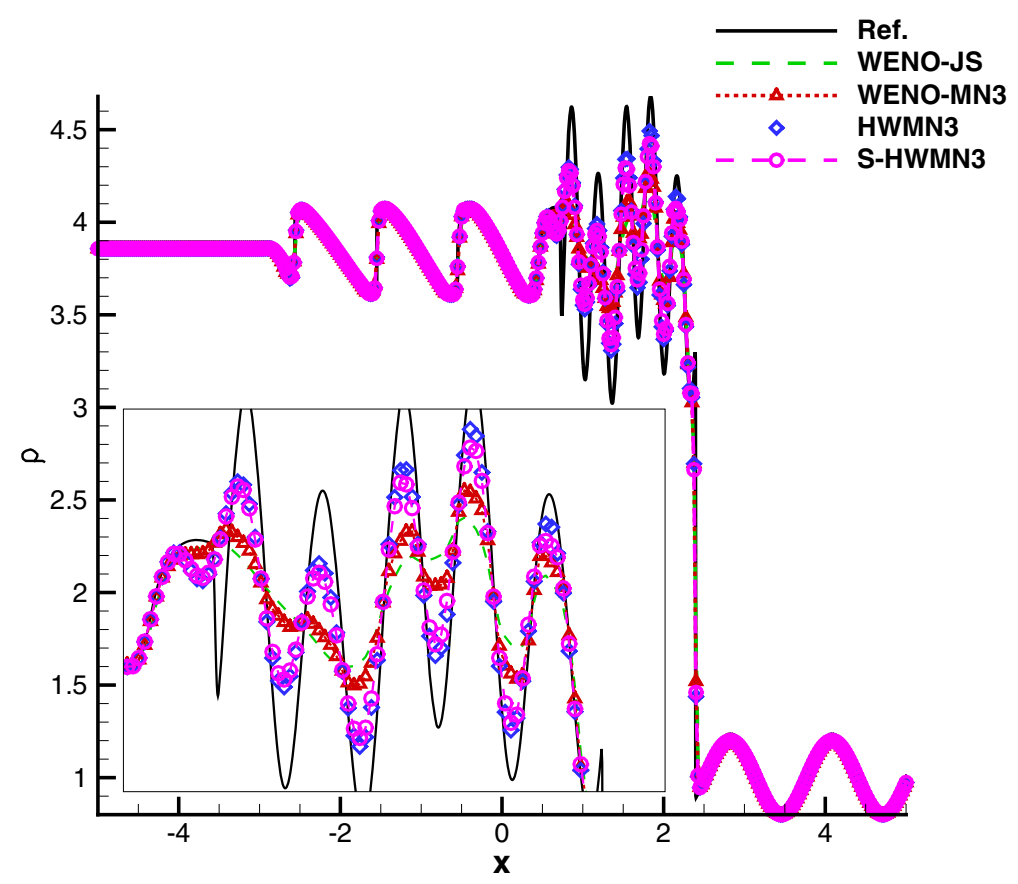

Fig. 12 Shu-Osher problem, $t=1.8, N=400$, using the WENO-JS, WENO-MN3, HWMN3 and S-HWMN3 schemes

\subsubsection{Case 5}

The first 1-D case is Shu-Osher problem [16], its initial condition is classically given as follows,

$$
(\rho, u, p)= \begin{cases}(3.857143,2.629369,31 / 3), & -5 \leq x<-4, \\ (1+0.2 \sin (5 x), 0,1), & -4 \leq x \leq 5 .\end{cases}
$$

Figure 12 gives the density distribution at $t=1.8$ with $N=400$. Both the hybrid schemes show apparent improvement over the others, and the HWMN3 scheme performs better than the S-HWMN3 scheme in the region with high frequency waves.

In fact, the initial condition Eq. (51) can be regarded as non-dimensional quantities by using certain reference values,

$$
L_{\text {ref }}=L_{0}, \quad \rho_{\text {ref }}=\rho_{0}, \quad U_{\text {ref }}=U_{0}
$$

Hence, if the reference values are taken as

$$
L_{r e f}=L_{0}, \quad \rho_{\text {ref }}=10 \rho_{0}, \quad U_{r e f}=10 U_{0}
$$

then the initial condition becomes as

$$
(\rho, u, p)= \begin{cases}(0.3857143,0.2629369,31 / 3000), & -5 \leq x<-4 \\ (0.1+0.02 \sin (5 x), 0,1 / 1000), & -4 \leq x \leq 5\end{cases}
$$

where $p_{r e f}=\rho_{r e f} \cdot U_{r e f}^{2}, t_{r e f}=L_{r e f} / U_{r e f}$. 


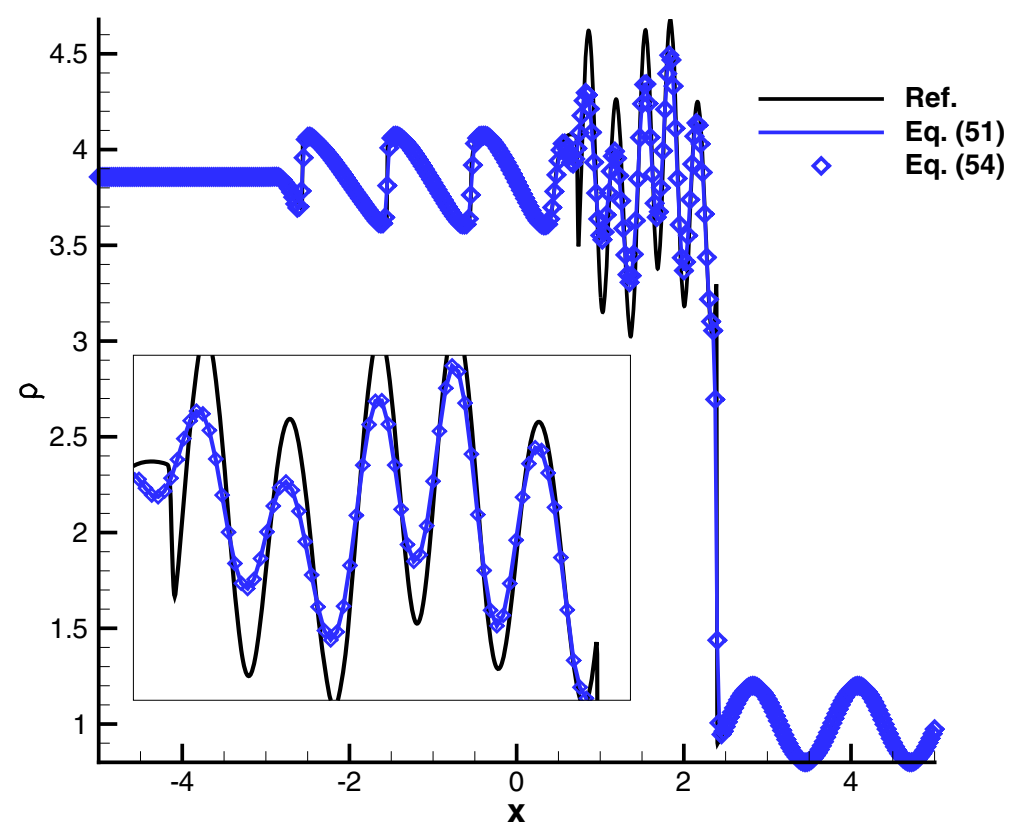

Fig. 13 Shu-Osher problem, using the HWMN3 scheme

The comparisons of densities computed by different schemes with the two initial conditions of Eqs. (51) and (54) are plotted in Figs. 13 and 14. Note that, in order to compare the results, all the numerical results are multiplied by its corresponding reference values, for example, the density of Eq. (54) plotted in corresponding figures is $\rho_{i}=10 \times \rho_{i}^{N}\left[\rho_{i}^{N}\right.$ is the numerical solutions of Eq. (54)], and the computational time is 10 times of that of Eq. (51). It can be seen that, the HWMN3 scheme can obtain similar results with different reference values, but WENO-NP3 and the weighted scheme with $\epsilon=\Delta x^{2}$ generate different solutions, although they can obtain good results. WENO-NN3 and WENO-NF3 are similar as WENO-NP3, thus their results are not shown for brevity.

\subsubsection{Case 6}

The second 1-D case is Lax problem [20]. Similar as Case 5, we also calculate the solutions with two kinds of initial conditions nondimensionalized by two sets of reference values,

$$
(\rho, u, p)= \begin{cases}(0.445,0.698,3.528) & -5 \leq x<0 \\ (0.5,0,0.571) & 0 \leq x \leq 5\end{cases}
$$

and

$$
(\rho, u, p)= \begin{cases}(0.0445,0.0698,3.528 / 1000) & -5 \leq x<0 \\ (0.05,0,0.571 / 1000) & 0 \leq x \leq 5\end{cases}
$$

The computational time is $t=1.3$ and $t=13$ for the initial conditions of Eqs. (55) and (56), respectively. The grid number is $N=200$. The density distribution of Eq. (55) is displayed in Fig. 15. All schemes can capture shocks well. 


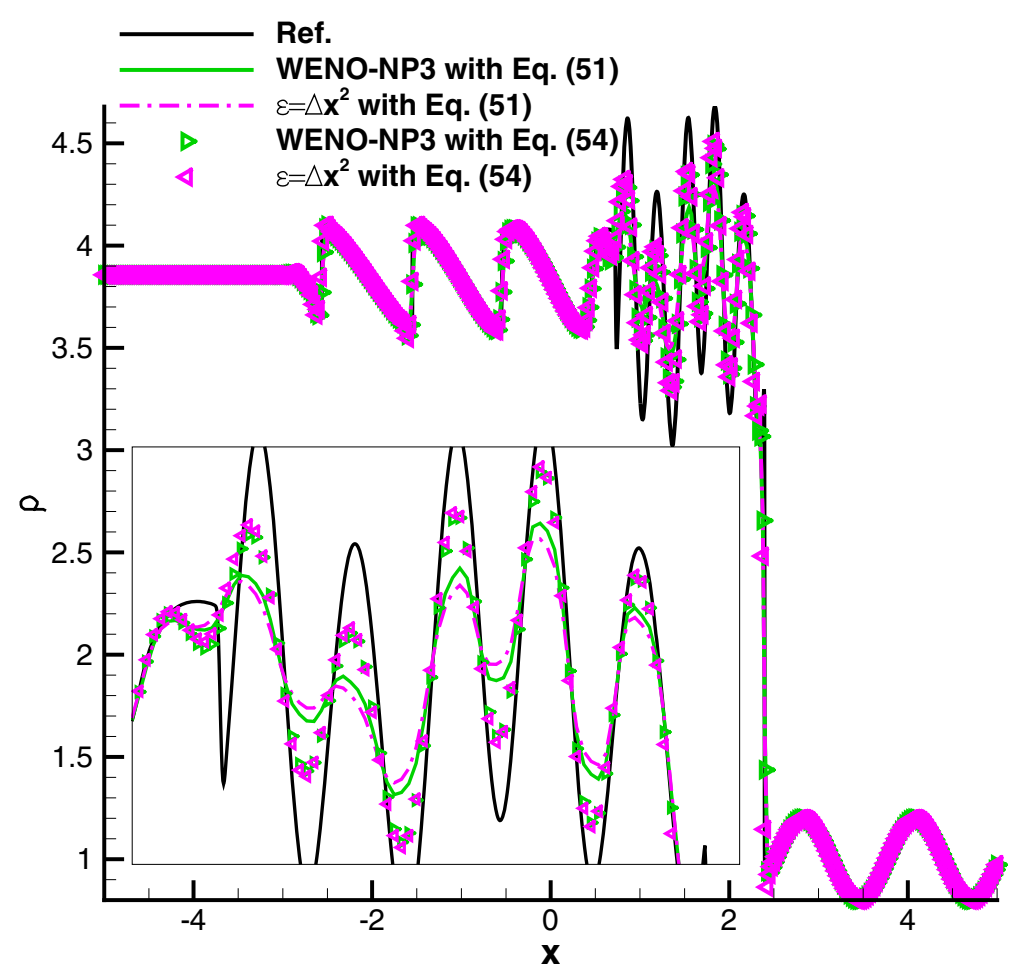

Fig. 14 Shu-Osher problem, using the WENO-NP3 and S-ESWENO schemes

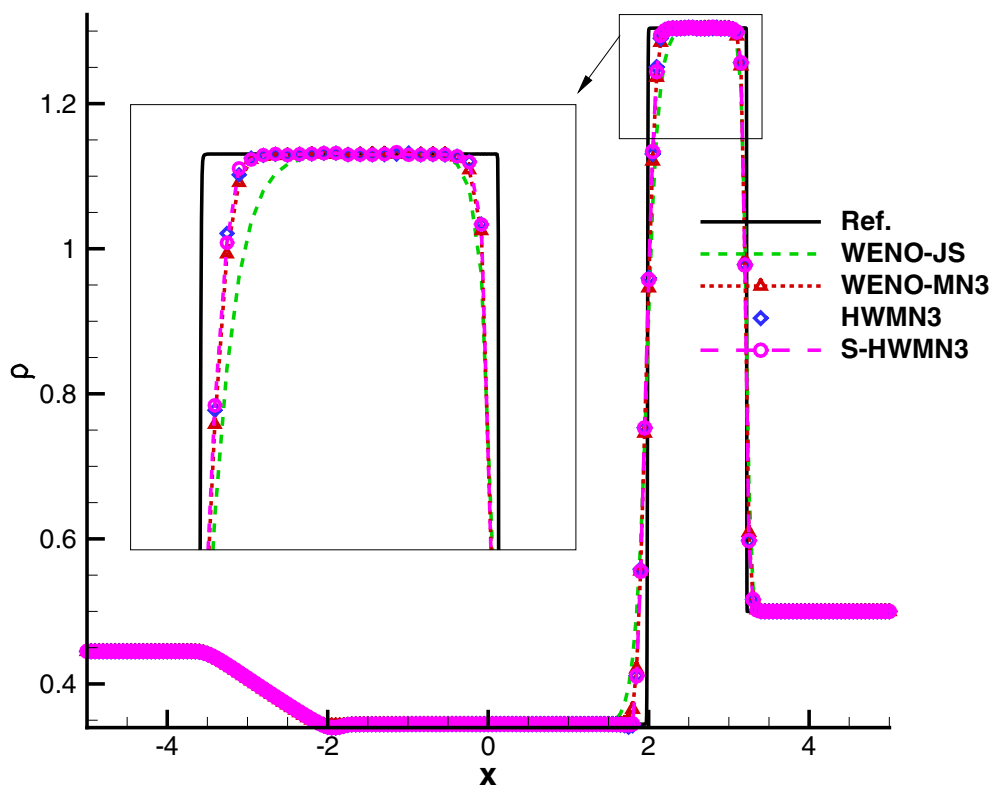

Fig. 15 Lax problem, $t=1.3, N=200$, using the WENO-JS, WENO-MN3, HWMN3 and S-HWMN3 schemes 


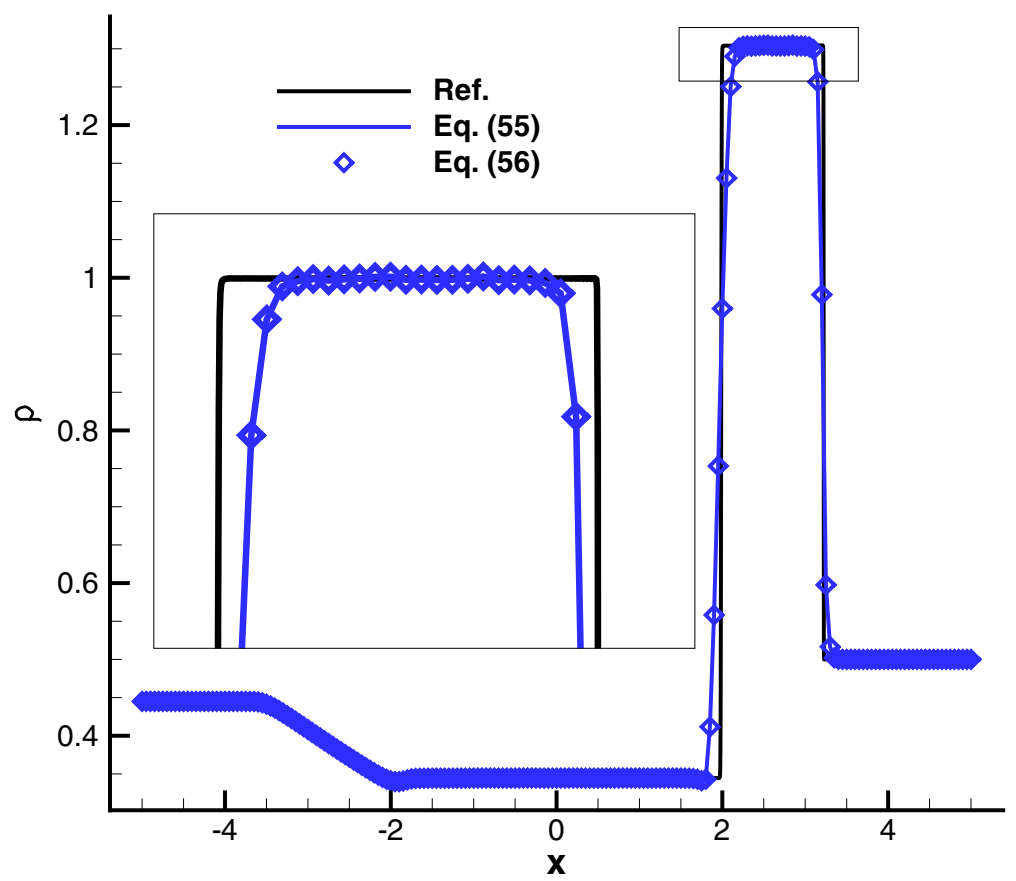

Fig. 16 Lax problem, using the HWMN3 scheme

The comparisons for different initial conditions of Eqs. (55) and (56) are shown in Figs. 16 and 17. Similar as Case 5, WENO-NP3 and the weighted scheme with $\epsilon=\Delta x^{2}$ obtain different results, even both schemes generate oscillations, especially for the case of Eq. (56).

\subsubsection{Case 7}

The last one is blast waves interaction problem [20], two non-dimensional initial conditions are

$$
(\rho, u, p)= \begin{cases}(1,0,1000) & 0 \leq x<0.1 \\ (1,0,0.001) & 0.1 \leq x<0.9 \\ (1,0,100) & 0.9 \leq x \leq 1\end{cases}
$$

and

$$
(\rho, u, p)= \begin{cases}(0.1,0,1) & 0 \leq x<0.1 \\ \left(0.1,0,10^{-6}\right) & 0.1 \leq x<0.9 \\ (0.1,0,0.1) & 0.9 \leq x \leq 1 .\end{cases}
$$

The computational time is $t=0.038$ and $t=0.38$ for the initial conditions of Eqs. (57) and (58), respectively. The grid number is $N=400$. The density distribution of Eq. (57) is displayed in Fig. 18. As we can see, near extrema, both the two hybrid schemes obtain more accurate solutions than WENO-MN3, and HWMN3 behaves better than S-HWMN3.

The comparisons for different initial conditions (see Figs. 19 and 20) show the similar conclusion as that in Case 5. 


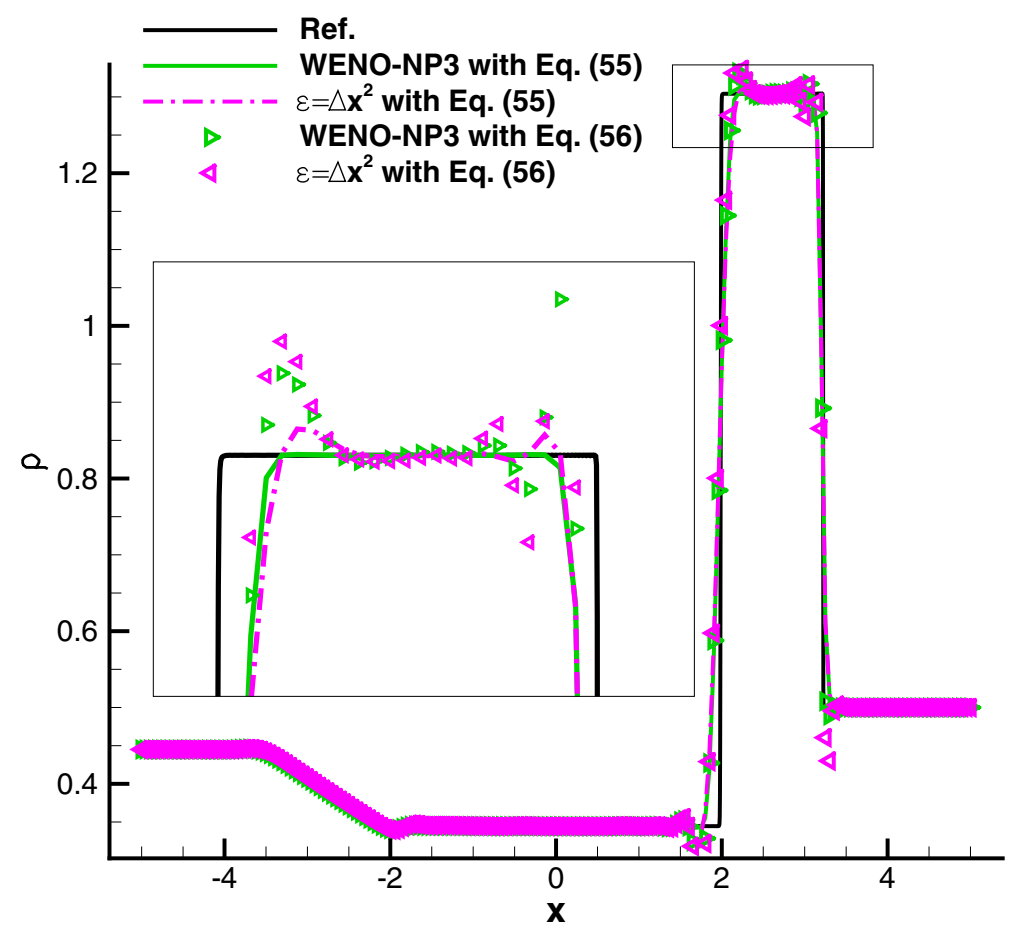

Fig. 17 Lax problem, using the WENO-NP3 and S-ESWENO schemes

\subsection{Two-Dimensional Euler Problems}

In this subsection, we further study the shock capturing ability and numerical dissipation of the hybrid schemes in the 2-D problems. The 2-D Euler equations are solved,

$$
\frac{\partial U}{\partial t}+\frac{\partial F}{\partial x}+\frac{\partial G}{\partial y}=0
$$

where the conserved variables $U$ and the inviscid flux vectors $F$ and $G$ are

$$
U=\left[\begin{array}{c}
\rho \\
\rho u \\
\rho v \\
E
\end{array}\right], \quad F=\left[\begin{array}{c}
\rho u \\
\rho u^{2}+p \\
\rho u v \\
E u+p u
\end{array}\right], \quad G=\left[\begin{array}{c}
\rho v \\
\rho u v \\
\rho v^{2}+p \\
E v+p v
\end{array}\right]
$$

The energy is given by

$$
E=\frac{p}{\gamma-1}+\frac{\rho}{2}\left(u^{2}+v^{2}\right)
$$




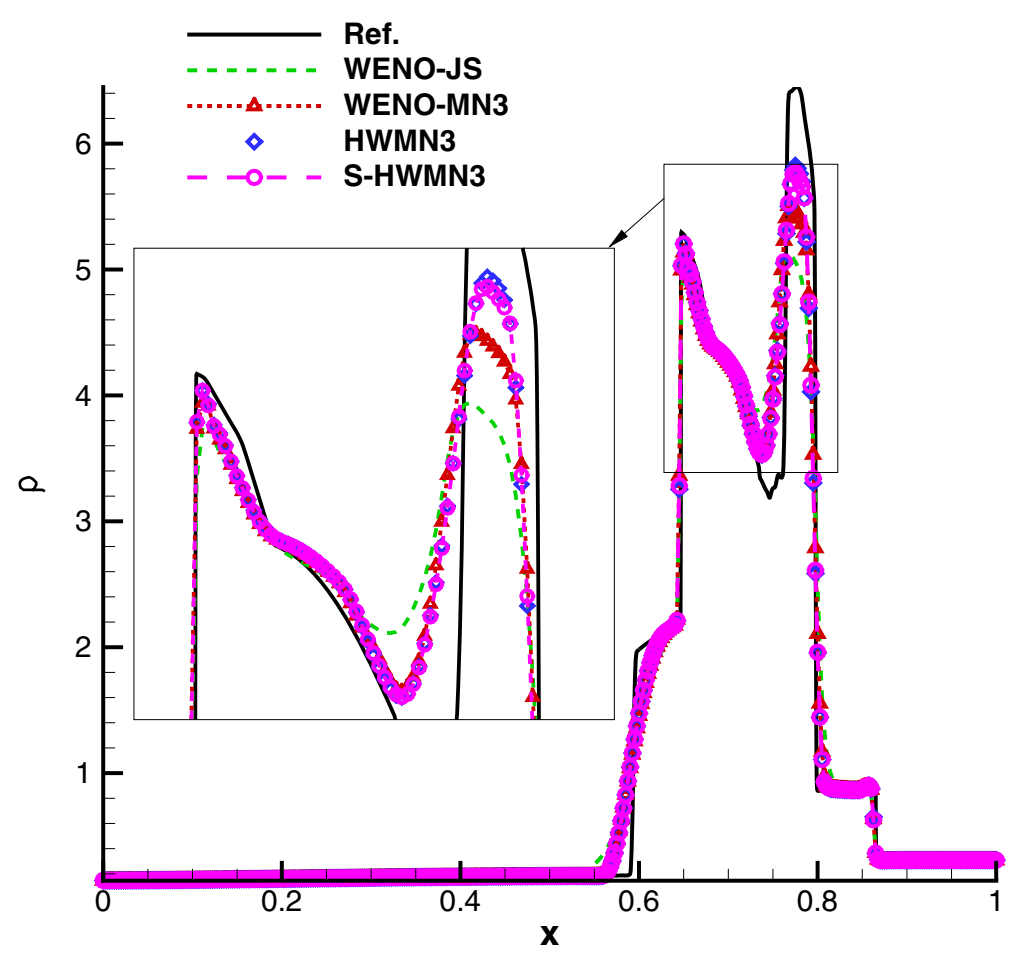

Fig. 18 Blast-waves interaction problem, $t=0.038, N=400$, using the WENO-JS, WENO-MN3, HWMN3 and S-HWMN3 schemes

The Roe [21] flux-splitting method is used for the inviscid convective fluxes, and the time step is taken as follows,

$$
\begin{aligned}
& \Delta t=\sigma \frac{\Delta t_{x} \Delta t_{y}}{\Delta t_{x}+\Delta t_{y}}, \\
& \left\{\begin{array}{l}
\Delta t_{x}=\frac{\Delta x}{\max _{i, j}\left(\left|u_{i, j}\right|+c_{i, j}\right)}, \\
\Delta t_{y}=\frac{\Delta y}{\max _{i, j}\left(\left|v_{i, j}\right|+c_{i, j}\right)},
\end{array}\right.
\end{aligned}
$$

where $\sigma$ is $C F L$ number, and 0.5 is used for all the tests below.

\subsubsection{Case 8}

This is a 2-D periodic vortex propagation problem [22,23] used to assess the numerical dissipation of different schemes. The vortex is described as a perturbation to the velocity $(u, v)$, temperature $(T=p / \rho)$ and entropy $(S=p / \rho)$ of the mean flow $(\rho, u, v, p)=$ $(1,1,1,1)$, 


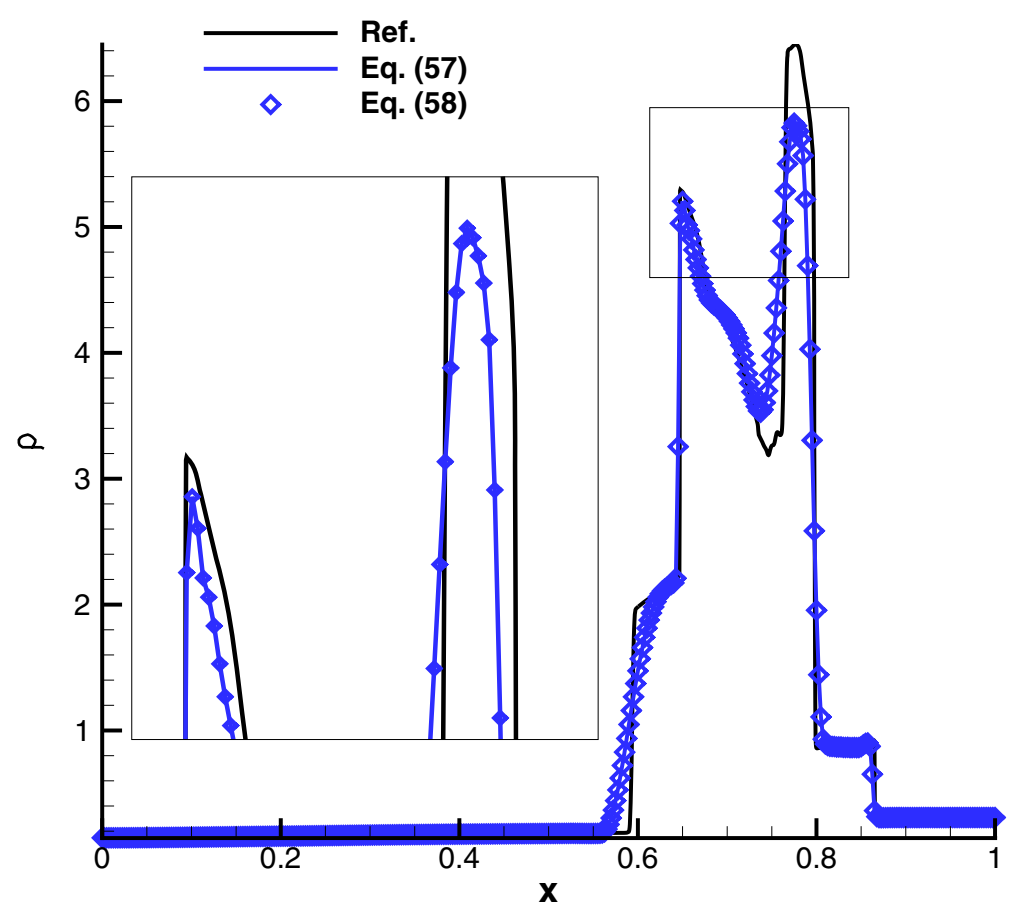

Fig. 19 Blast-waves interaction problem, using the HWMN3 scheme

$$
\left\{\begin{array}{l}
\hat{u}=-\frac{\epsilon e^{\left(1-r^{2}\right) / 2}}{2 \pi} \bar{y} \\
\hat{v}=\frac{\epsilon e^{\left(1-r^{2}\right) / 2}}{2 \pi} \bar{x} \\
\hat{T}=-\frac{(\gamma-1) \epsilon^{2} e^{\left(1-r^{2}\right)}}{8 \gamma \pi^{2}} \\
\hat{S}=0
\end{array}\right.
$$

where $(\bar{x}, \bar{y})=(x-5, y-5), r^{2}=\bar{x}^{2}+\bar{y}^{2}$, and the vortex strength $\epsilon=0.5$. The computational domain is $[0,10] \times[0,10]$, and periodic boundary condition is used in both directions. The results are carried out until $t=200$ with a grid of $100 \times 100$. Figure 21 shows the density contours. Figures 22 and 23 show the density distribution along $x$ axis at $y=5$, and the evolution of kinetic energy $E_{k}$, respectively. $E_{k}$ is calculated by $E_{k}=\sum_{i, j=(1,1)}^{N, M} \rho_{i, j}\left(u_{i, j}^{2}+v_{i, j}^{2}\right)$, where $N$ and $M$ are the total cells in $x$ and $y$ direction of the grid, respectively. Figures 21, 22 and 23 show that, the two hybrid schemes can preserve the shape of vortex as good as the Up3 scheme, they have almost the same dissipation as Up3.

\subsubsection{Case 9}

This is a 2-D Riemann problem [24] used to test the shock capturing capability of the hybrid schemes for calculating shock/shock interaction problems. It is solved on a unit square with initial condition, 


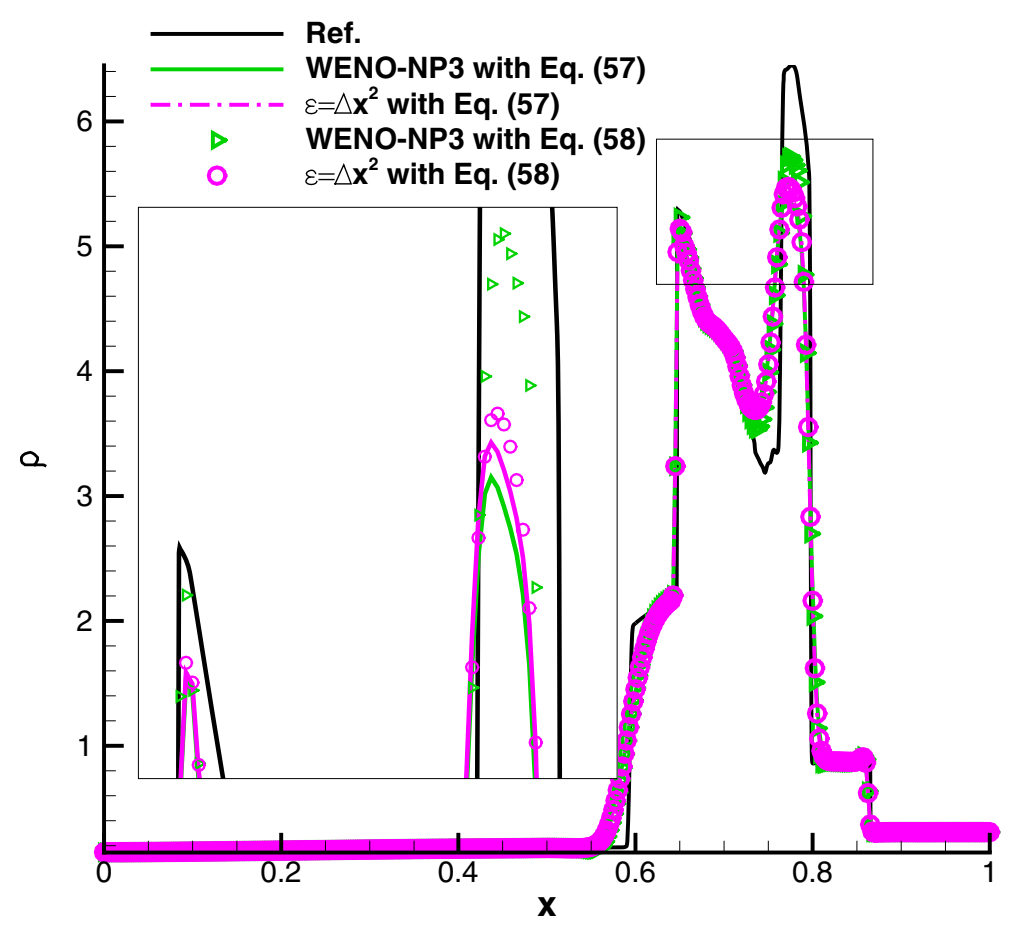

Fig. 20 Blast-waves interaction problem, using the WENO-NP3 and S-ESWENO schemes

$$
(\rho, u, v, p)= \begin{cases}(1.0,-0.75,-0.5,1.0), & 0.5 \leq x \leq 1,0.5 \leq y \leq 1 \\ (2.0,-0.75,0.5,1.0), & 0 \leq x<0.5,0.5 \leq y \leq 1 \\ (1.0,0.75,0.5,1.0), & 0 \leq x<0.5,0 \leq y<0.5 \\ (3.0,0.75,-0.5,1.0), & 0.5 \leq x \leq 1,0 \leq y<0.5\end{cases}
$$

The solution at $t=0.23$ is solved with a grid of $400 \times 400$. The reference is calculated by WENO-JS with a grid of $800 \times 800$. Figure 24 shows the density contours of the reference solution and the results of different schemes. As shown in the figure, since the hybrid schemes switch to the WENO-MN3 scheme on discontinuous stencils, the hybrid schemes have similar shock-capturing capability as the WENO-MN3 scheme.

\subsubsection{Case 10}

The 2-D shock/vortex interaction problem describes an interaction between a stationary shock and a vortex [3]. The computational domain is taken to be $[0,2] \times[0,1]$. A stationary Mach 1.1 shock is positioned at $x=0.5$ and normal to the $x$-axis. Its left state is $(\rho, u, v, p)=$ $(1,1.1 \sqrt{\gamma}, 0,1)$. A small vortex is superimposed to the flow on the left of the shock and centered at $\left(x_{c}, y_{c}\right)=(0.25,0.5)$. The vortex is described as a perturbation to the velocity $(u, v)$, temperature $(T=p / \rho)$ and entropy $S=\ln \left(p / \rho^{\gamma}\right)$ of the mean flow, 


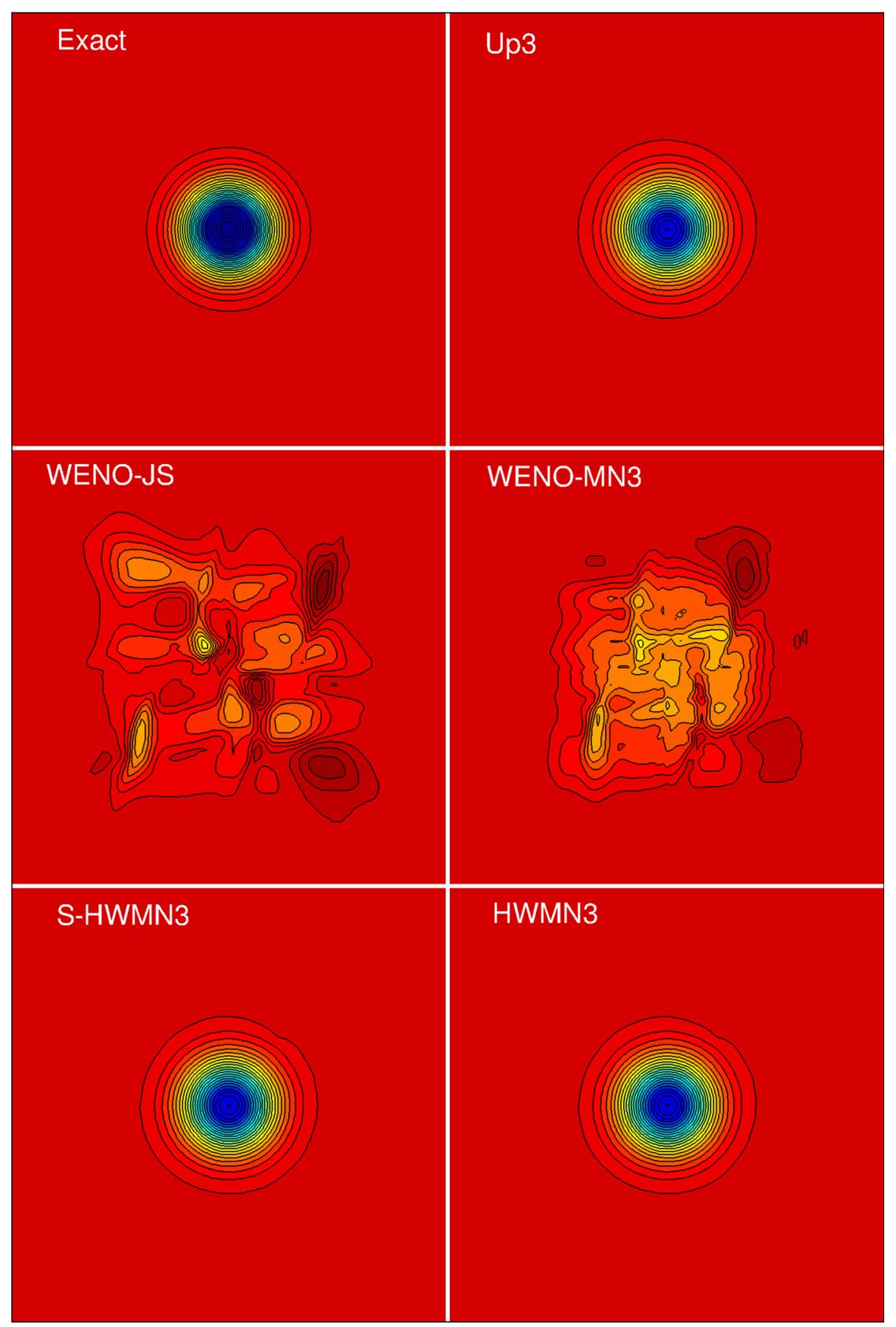

Fig. 21 Density contours of vortex propagation problem, 30 contours from 0.9941 to 1.0007 


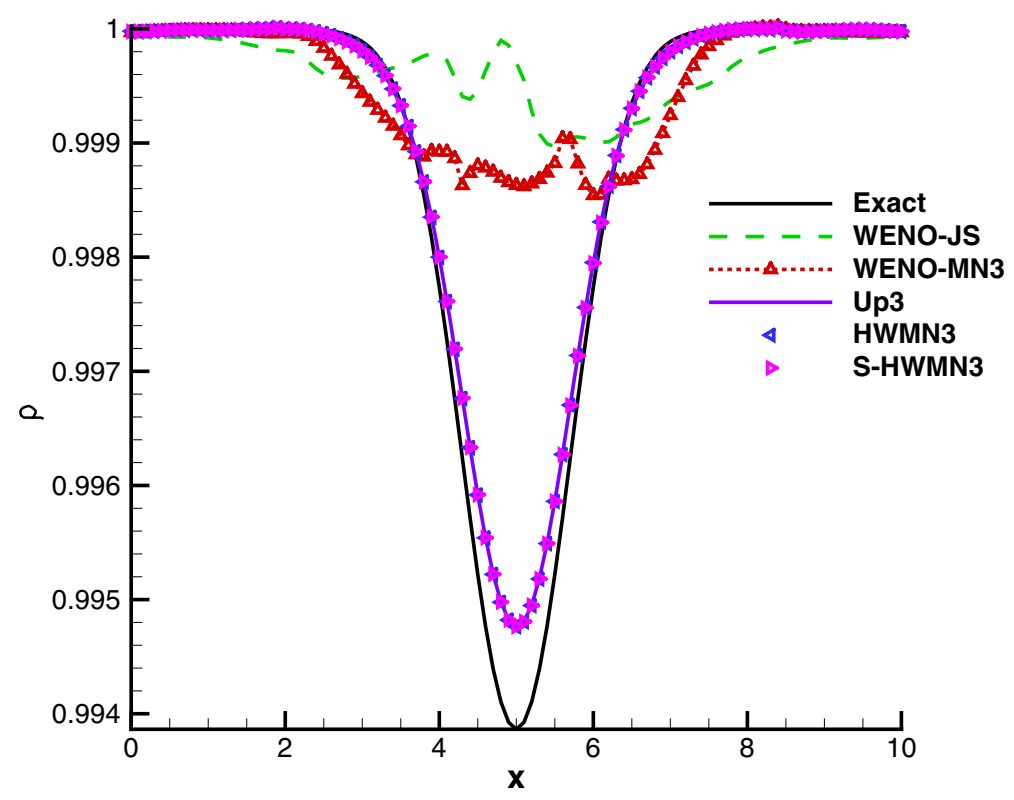

Fig. 22 Density distribution along $\mathrm{y}=5$

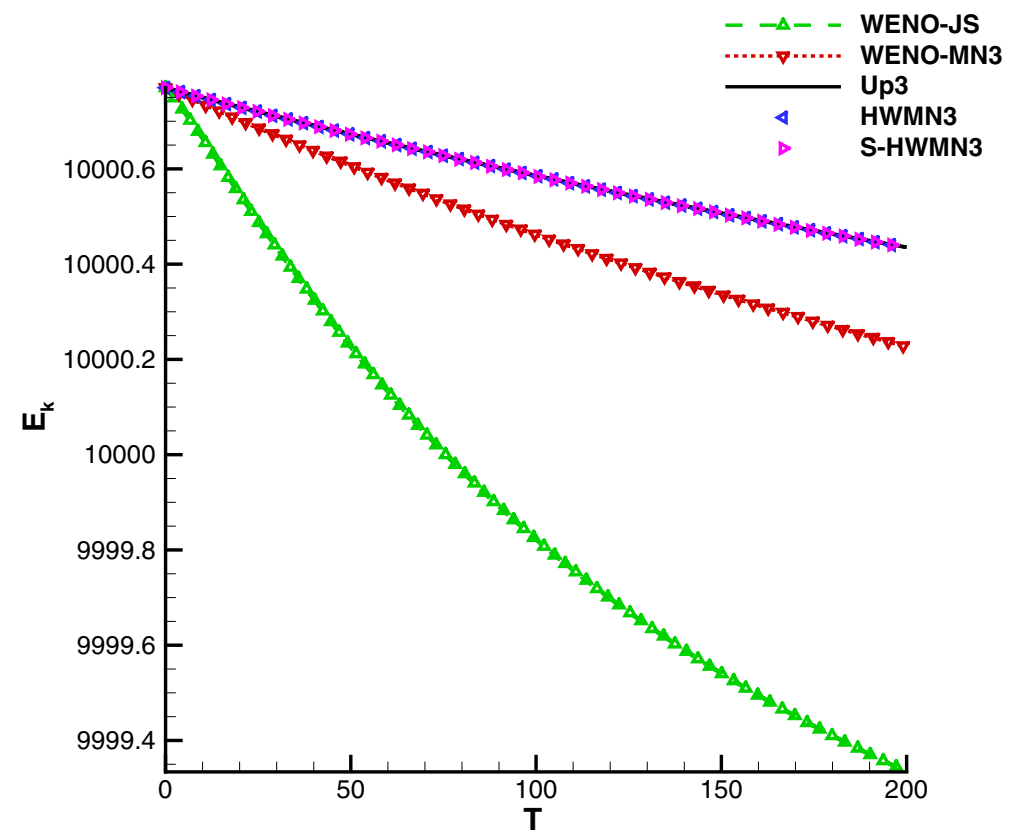

Fig. 23 Evolution of $E_{k}$ of vortex propagation problem until $t=200$ 


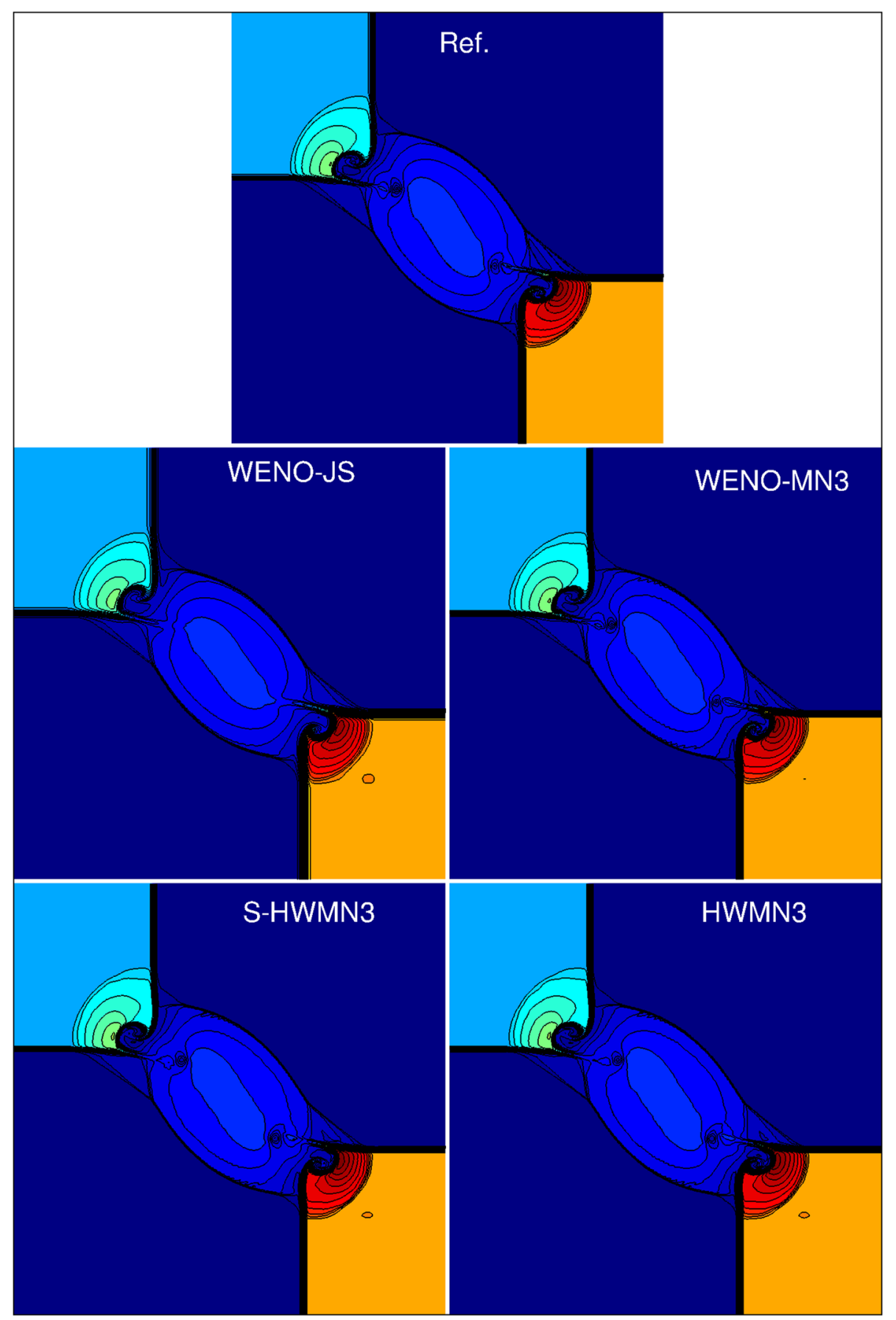

Fig. 24 Density contours of Riemann problem, 30 contours from 1.089 to 3.926 


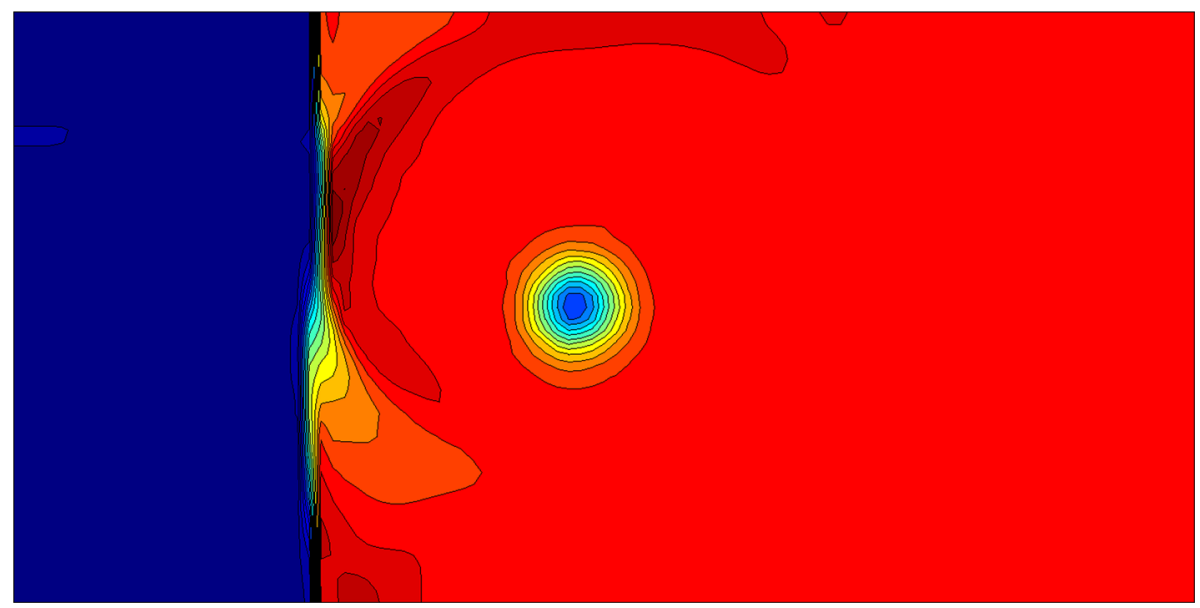

Fig. 25 Pressure contours of shock/vortex interaction problem, computed by the HWMN3 scheme, 20 contours from 1.005 to 1.297

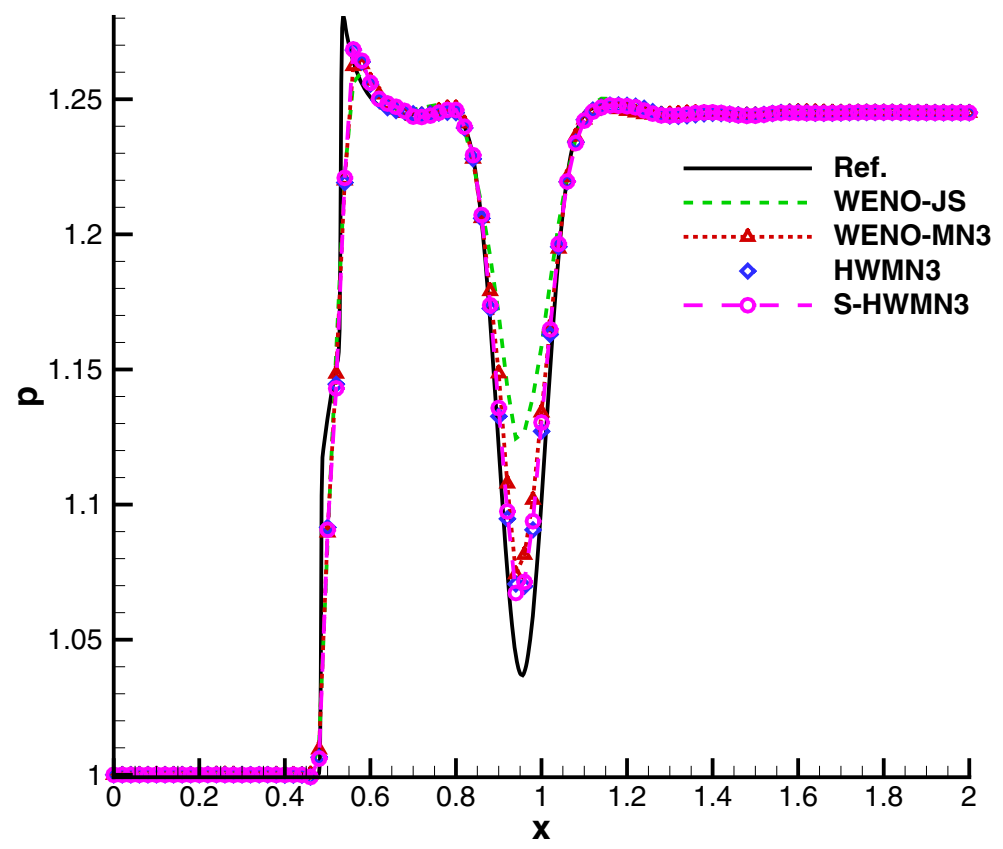

Fig. 26 Pressure distributed along $y=0.5$ of shock/vortex interaction problem

$$
\left\{\begin{array}{l}
\hat{u}=\epsilon \tau e^{a\left(1-\tau^{2}\right)} \sin \theta \\
\hat{v}=-\epsilon \tau e^{a\left(1-\tau^{2}\right)} \cos \theta \\
\hat{T}=-\frac{(\gamma-1) \epsilon^{2} e^{2 a\left(1-\tau^{2}\right)}}{4 a \gamma} \\
\hat{S}=0
\end{array}\right.
$$




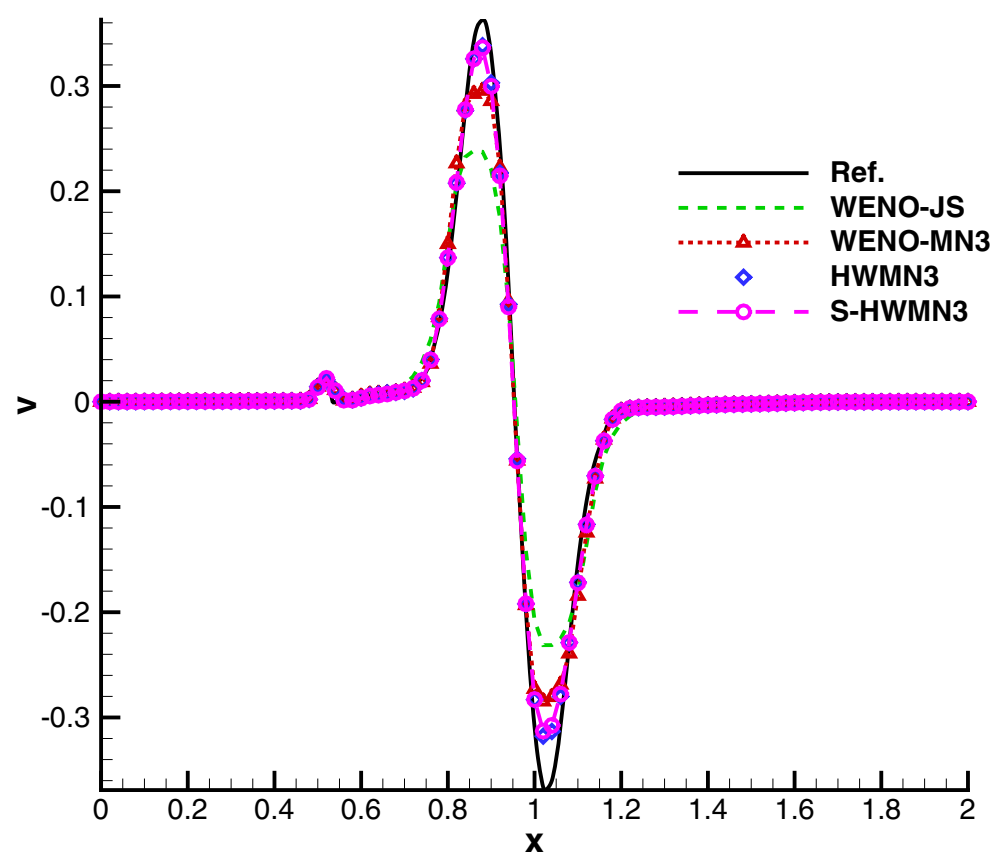

Fig. 27 Velocity $v$ distributed along $y=0.5$ of shock/vortex interaction problem

where, $\tau=r / r_{c}, r=\sqrt{\left(x-x_{c}\right)^{2}+\left(y-y_{c}\right)^{2}}, \epsilon=0.3, r_{c}=0.05$ and $a=0.204$ are the same as in Refs. [3,25]. The upper and lower boundaries are set to be reflective. The results at $t=0.6$ with a grid of $100 \times 50$ are solved. Figure 25 shows the pressure contours calculated by the HWMN3 scheme. Figures 26 and 27 plot the pressure and velocity $v$ distribution along the center line of $y=0.5$, respectively. The result obtained by WENO-JS with a refined mesh of $2000 \times 1000$ is given as the reference solution. It can be seen that the pressure distribution of the WENO-MN3 and the two hybrid schemes is almost the same, but the velocity distribution around the vortex is improved apparently by the two hybrid schemes.

\section{Conclusion Remarks}

The analyses in this paper show that, one reason for the accuracy losing of the third-order WENO schemes is that the local smoothness indicators cannot correctly treat the threepoint stencil containing non-nodal critical point. In order to overcome this shortcoming, a discontinuity-detecting method for a four-point stencil is proposed and applied to construct a hybrid scheme (denoted by HWMN3) of the WENO-MN3 scheme combining with a third-order upstream scheme. As the analyses shown, combining the discontinuity-detecting methods for the three-point (Eq. 40) and four-point (Eq. 39) stencils, the three-point stencil containing a non-nodal critical point can be effectively detected, hence it can be correctly treated in the HWMN3 scheme. The numerical results show that the HWMN3 scheme can recover to full third-order accuracy for smooth solutions and maintain the ENO property simultaneously. 
A simplified hybrid scheme (denoted by S-HWMN3) is also suggested. Although the SHWMN3 scheme may lose its accuracy partly, its performances are much better than those third-order WENO schemes and comparable with the HWMN3 scheme. In addition, the SHWMN3 scheme is less computational cost than the HWMN3 scheme, so S-HWMN3 can also be a good candidate scheme.

Acknowledgements This research work was supported by the NKRDPC 2016YFA0401200, SCP No. TZ2016002, NSAF U1530145, NSFC Nos. 11872067 and 91852203.

\section{References}

1. Liu, X.D., Osher, S., Chan, T.: Weighted essentially non-oscillatory schemes. J. Comput. Phys. 115, 200-212 (1994)

2. Harten, A., Engquist, B., Osher, S., Chakravarthy, S.: Uniformly high order essentially non-oscillatory schemes III. J. Comput. Phys. 71, 231-303 (1987)

3. Jiang, G.S., Shu, C.W.: Efficient implementation of weighted ENO schemes. J. Comput. Phys. 126, 202-228 (1996)

4. Balsara, D.S., Shu, C.W.: Monotonicity preserving weighted essentially non-oscillatory schemes with increasingly high order of accuracy. J. Comput. Phys. 160, 405-452 (2000)

5. Gerolymos, G., Senechal, D., Vallet, I.: Very-high-order WENO schemes. J. Comput. Phys. 228, 84818524 (2009)

6. Liu, S., Shen, Y., Chen, B., Zeng, F.: Novel local smoothness indicators for improving the third-order WENO scheme. Int. J. Numer. Methods Fluids 87, 51-69 (2018)

7. Yamaleev, N.K., Carpenter, M.H.: Third-order energy stable WENO scheme. J. Comput. Phys. 228, 3025-3047 (2009)

8. Wu, X., Zhao, Y.: A high-resolution hybrid scheme for hyperbolic conservation laws. Int. J. Numer. Methods Fluids 78, 162-187 (2015)

9. Wu, X., Lian, J., Zhao, Y.: A new smoothness indicator for third-order WENO scheme. Int. J. Numer. Methods Fluids 81, 451-459 (2016)

10. Gande, N.R., Rathod, Y., Rathan, S.: Third-order WENO scheme with a new smoothness indicator. Int. J. Numer. Methods Fluids 85, 90-112 (2017)

11. Xu, W., Wu, W.: An improved third-order weighted essentially non-oscillatory scheme achieving optimal order near critical points. Comput. Fluids 162, 113-125 (2018)

12. Xu, W., Wu, W.: An improved third-order WENO-Z scheme. J. Sci. Comput. 75, 1808-1841 (2018)

13. Acker, F., de Borges, R.B.R., Costa, B.: An improved WENO-Z scheme. J. Comput. Phys. 313, 726-753 (2016)

14. Henrick, A.K., Aslam, T.D., Powers, J.M.: Mapped weighted essentially non-oscillatory schemes: achieving optimal order near critical points. J. Comput. Phys. 207, 542-567 (2005)

15. Shen, Y., Zha, G.: Generalized finite compact difference scheme for shock/complex flowfield interaction. J. Comput. Phys. 230, 4419-4436 (2011)

16. Shu, C.W., Osher, S.: Efficient implementation of essentially non-oscillatory shock-capturing schemes. J. Comput. Phys. 77, 439-471 (1988)

17. Szirtes, T., Rozsa, P.: Applied dimensional analysis and modeling (second edition). Appl. Dimens. Anal. Model. 25, 99-132 (2007)

18. Najiafi-Yazdi, A., Mongeau, L.: A low-dispersion and low-dissipation implicit Runge-Kutta scheme. J. Comput. Phys. 233, 315-323 (2013)

19. Shu, C.W.: Essentially non-oscillatory and weighted essentially non-oscillatory schemes for hyperbolic conservation laws. ICASE Report 97-65 (1997)

20. Borges, R., Carmona, M., Costa, B., Don, W.S.: An improved weighted essentially non-oscillatory scheme for hyperbolic conservation laws. J. Comput. Phys. 227, 3191-3211 (2008)

21. Roe, P.L.: Approximate Riemann solvers, parameter vectors, and difference schemes. J. Comput. Phys. 43, 357-372 (1981)

22. Sun, Y.Z., Wang, Z.: Evaluation of discontinuous Galerkin and spectral volume methods for scalar and system conservation laws on unstructured grids. Int. J. Numer. Methods Fluids 45, 819-838 (2004)

23. Davoudzadeh, F., McDonald, H., Thopson, B.: Accuracy evaluation of unsteady CFD numerical schemes by vortex preservation. Comput. Fluids 24, 883-895 (1995) 
24. Kurganov, A., Tadmor, E.: Solution of two-dimensional Riemann problems for gas dynamics without Riemann problem solvers. Numer. Methods Partial Differ. Equ. 18, 584-608 (2002)

25. Shen, Y.Q., Yang, G.W.: Hybrid finite compact-WENO schemes for shock calculation. Int. J. Numer. Methods Fluids 53, 531-560 (2007)

Publisher's Note Springer Nature remains neutral with regard to jurisdictional claims in published maps and institutional affiliations. 